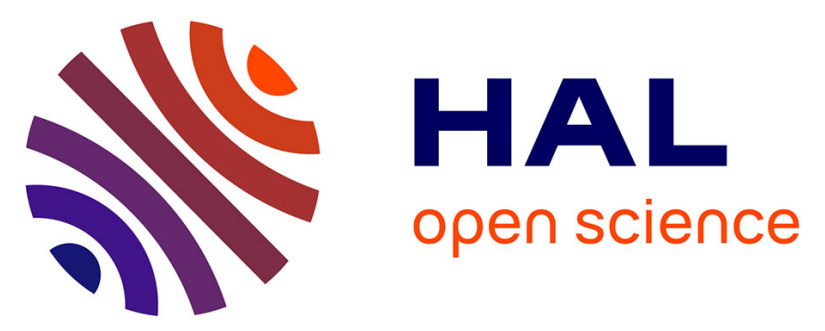

\title{
ADAMTSL5 is an epigenetically activated gene underlying tumorigenesis and drug resistance in hepatocellular carcinoma
}

Maria Arechederra, Sehrish Bazai, Ahmed Abdouni, Celia Sequera, Timothy Mead, Sylvie Richelme, Fabrice Daian, Stéphane Audebert, Rosanna Dono, Anthony Lozano, et al.

\section{To cite this version:}

Maria Arechederra, Sehrish Bazai, Ahmed Abdouni, Celia Sequera, Timothy Mead, et al.. ADAMTSL5 is an epigenetically activated gene underlying tumorigenesis and drug resistance in hepatocellular carcinoma. Journal of Hepatology, 2021, 10.1016/j.jhep.2020.11.008 . hal-03049722

\section{HAL Id: hal-03049722 \\ https://hal.science/hal-03049722}

Submitted on 10 Dec 2020

HAL is a multi-disciplinary open access archive for the deposit and dissemination of scientific research documents, whether they are published or not. The documents may come from teaching and research institutions in France or abroad, or from public or private research centers.
L'archive ouverte pluridisciplinaire $\mathbf{H A L}$, est destinée au dépôt et à la diffusion de documents scientifiques de niveau recherche, publiés ou non, émanant des établissements d'enseignement et de recherche français ou étrangers, des laboratoires publics ou privés. 


\section{Journal of Hepatology}

\section{ADAMTSL5 is an epigenetically activated gene that confers tumorigenic properties and drug resistance in hepatocellular carcinoma \\ --Manuscript Draft--}

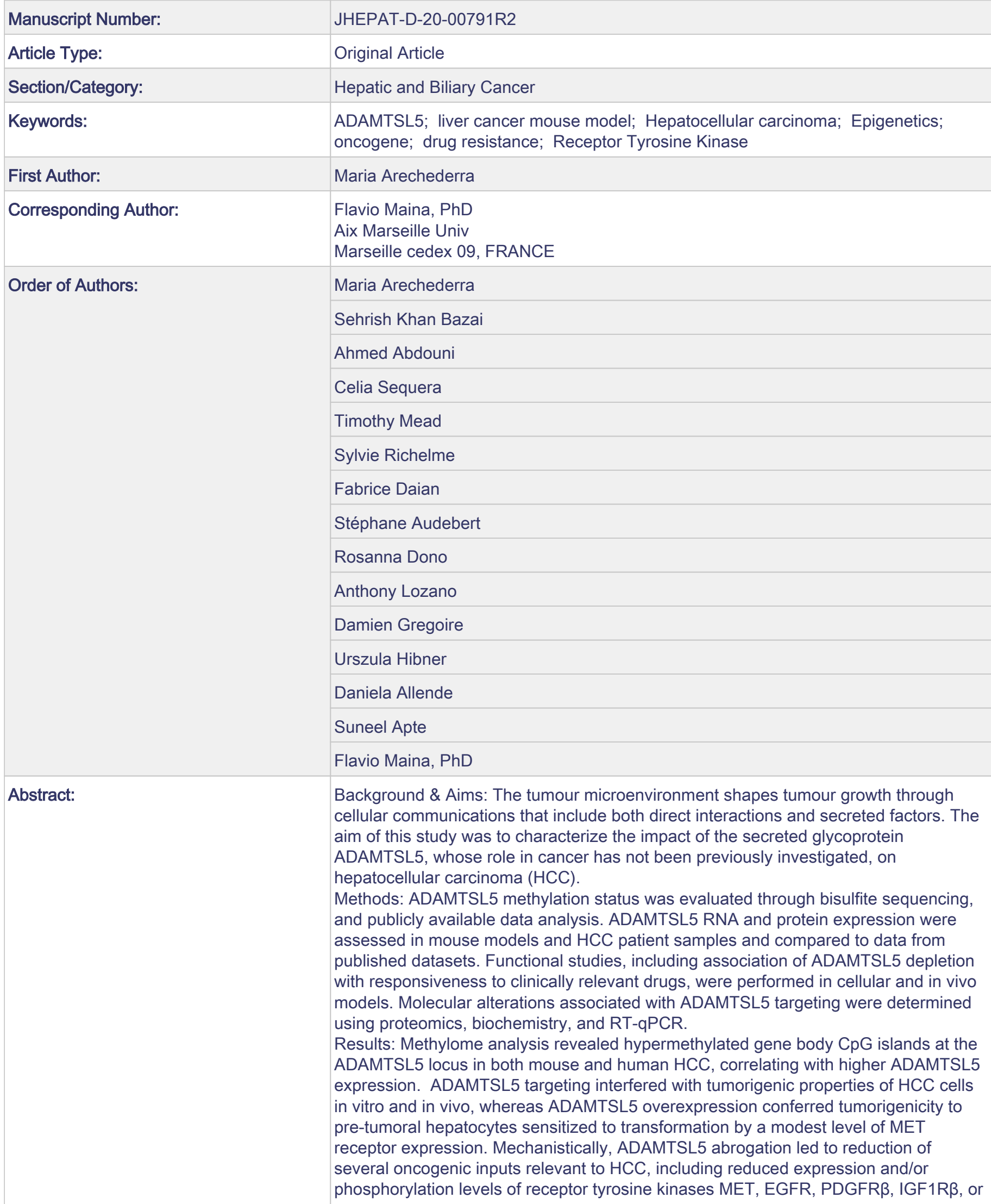


FGFR4. This phenotype was associated with significantly increased sensitivity of HCC cells to clinically relevant drugs, namely Sorafenib, Lenvatinib, Regorafenib. Moreover, ADAMTSL5 depletion drastically increased expression of AXL, accompanied by a sensitization to Bemcentinib.

Conclusions: Our results point to a role for ADAMTSL5 in maintaining the function of key oncogenic signalling pathways, suggesting that it may act as a master regulator of tumorigenicity and drug resistance in $\mathrm{HCC}$.

Response to Reviewers: 


\section{ADAMTSL5 is an epigenetically activated gene that confers tumorigenic} properties and drug resistance in hepatocellular carcinoma

Maria Arechederra ${ }^{1, \dagger}$, Sehrish K. Bazai ${ }^{1, \uparrow}$, Ahmed Abdouni ${ }^{1, \dagger}$, Celia Sequera ${ }^{1, \dagger}$, Timothy J. Mead $^{2}$, Sylvie Richelme ${ }^{1}$, Fabrice Daian ${ }^{1}$, Stéphane Audebert ${ }^{3}$, Rosanna Dono ${ }^{1}$, Anthony Lozano $^{4}$, Damien Gregoire ${ }^{4}$, Urszula Hibner ${ }^{4}$, Daniela S. Allende ${ }^{5}$, Suneel S. Apte ${ }^{2}$, and Flavio Maina $^{1, *}$

1 Aix Marseille Univ, CNRS, Developmental Biology Institute of Marseille (IBDM), UMR7288, Parc Scientifique de Luminy, Marseille (France).

${ }^{2}$ Department of Biomedical Engineering, Cleveland Clinic Lerner Research Institute, Cleveland, OH 44195 (USA).

3 Aix-Marseille Univ, CRCM, Marseille Proteomics, INSERM, CNRS, Institut PaoliCalmettes, Marseille, France.

${ }^{4}$ Institut de Génétique Moléculaire de Montpellier, Univ Montpellier, CNRS, Montpellier, France.

${ }^{5}$ Pathology Department, Pathology and Laboratory Medicine Institute, Cleveland Clinic, Cleveland, OH, 44195 (USA).

${ }^{\dagger}$ These authors contributed equally to this work.

*Corresponding author (flavio.maina@univ-amu.fr) 


\section{Contact information of the corresponding author}

Flavio Maina, Ph.D.

IBDM (Developmental Biology Institute of Marseille)

163 Avenue de Luminy, case 907 - 13009 Marseille (France)

Phone: +33(0)4 91269769

flavio.maina@univ-amu.fr

Key words: ADAMTSL5; liver cancer mouse model; hepatocellular carcinoma; epigenetics; oncogene; drug resistance; Receptor Tyrosine Kinase

Electronic word count: abstract, references, tables, and figure legends: 6598

\section{Number of figures and tables: 8 Figures}

Conflict of interest. The authors M.A., T.J.M., R.D., S.S.A., and F.M. are inventors of the patent: "Method and kit for diagnosing and for treatment of a cancer based on the overexpression of the ADAMTSL5 gene".

Financial support. This work was funded by FdF (Fondation de France; 2014_00051580 and 2016_00067080), ARC (Association pour la Recherche sur le Cancer; PJA20181208172), GEFLUC - Les Entreprises contre le Cancer, and SATT Sud-Est to F.M. This study was partly supported by research funding from Institut National du Cancer, Règion Provence-Alpes-Côte d'Azur, and Canceropôle Provence-Alpes-Côte d'Azur to F.M. S.S.A. received support from the Allen Distinguished Investigator Program, through support made by The Paul G. Allen Frontiers Group and the American Heart Association (17 DIA33820024); D.S.A. and S.S.A 
received support from Velosano. M.A. was supported by a FdF fellowship. S.K.B. was supported by the Higher Education Commission (HEC) of Pakistan. A.A. was supported with a grant from SATT Sud-Est. C.S. was supported by a FRM fellowship. A.L. was supported by an ARC fellowship. The contribution of the Region Provence-Alpes-Côte d'Azur and of the Aix-Marseille Univ to the IBDM animal facility is acknowledged. Proteomic analyses were performed at the mass spectrometry facility of Marseille Proteomics supported by IBISA (Infrastructures Biologie Santé et Agronomie), Plateforme Technologique Aix-Marseille, Canceropôle PACA, Région Sud Provence-Alpes-Côte d'Azur, Fonds Européen de Développement Régional (FEDER) and Plan Cancer. The funders had no role in study design, data collection and analysis, decision to publish, or preparation of the manuscript.

\section{Author contributions}

M.A.: performed the majority of the experiments with mouse HCC, methylation studies, computational work with human HCC databases, RT-qPCR on human cohort \#3 samples, data analysis, and interpretation; provided input on studies and contributed to write the manuscript.

S.K.B.: generated lentivirus, contributed to molecular, biochemical, xenograft, and functional studies with mouse and human HCC cells.

A.A.: prepared samples for secretome studies, contributed to molecular, biochemical, and functional validation of outcomes.

C.S.: performed analysis on human HCC cells, contributed to molecular, functional, and methylation studies, performed bioinformatics analysis of human HCC databases, provided input on studies.

T.J.M.: performed analyses on Alb-R26Met and human HCC samples, data analysis, and interpretation; provided input on studies and on the manuscript. 
S.R.: generated and characterized immorto-R26Met hepatocytes; performed tumour sphere assays, immunostaining of cells, and Decitabine treatment of $A l b-R 26^{M e t}$ mice; contributed to xenograft and orthotopic studies.

F.D.: contributed to computational work with human databases, data analysis, and interpretation.

S.A.: performed and analysed secretome studies.

R.D.: contributed to establishing the $A l b-R 26^{M e t}$ mouse model, to the generation of lentivirus, to molecular and morphological studies with HCC cells, data analysis and interpretation; provided input on studies.

D.G.: performed and provided input on orthotopic studies, generated $N$-Ras ${ }^{G 12 D}$ :p53null mouse tumours, prepared human cohort \#4 samples, contributed to write the manuscript.

A.L.: performed orthotopic studies, RT-qPCR on N-RasG12D:p53null mouse tumours and on human cohort \#4 samples.

U.H.: provided input on studies and contributed to write the manuscript.

D.S.A.: contributed to studies with human HCC samples (selection of HCC cases, controls, interpretation of staining).

S.S.A.: designed and supervised studies on human HCC samples; provided input on studies; contributed to interpret data and to write the manuscript.

F.M.: designed the study, contributed to experimental work, analysed and interpreted data, ensured financial support, and wrote the manuscript. 


\begin{abstract}
Background \& Aims: The tumour microenvironment shapes tumour growth through cellular communications that include both direct interactions and secreted factors. The aim of this study was to characterize the impact of the secreted glycoprotein ADAMTSL5, whose role in cancer has not been previously investigated, on hepatocellular carcinoma (HCC).
\end{abstract}

Methods: ADAMTSL5 methylation status was evaluated through bisulfite sequencing, and publicly available data analysis. ADAMTSL5 RNA and protein expression were assessed in mouse models and HCC patient samples and compared to data from published datasets. Functional studies, including association of ADAMTSL5 depletion with responsiveness to clinically relevant drugs, were performed in cellular and in vivo models. Molecular alterations associated with ADAMTSL5 targeting were determined using proteomics, biochemistry, and RT-qPCR.

Results: Methylome analysis revealed hypermethylated gene body $\mathrm{CpG}$ islands at the ADAMTSL5 locus in both mouse and human HCC, correlating with higher ADAMTSL5 expression. ADAMTSL5 targeting interfered with tumorigenic properties of HCC cells in vitro and in vivo, whereas ADAMTSL5 overexpression conferred tumorigenicity to pre-tumoral hepatocytes sensitized to transformation by a modest level of MET receptor expression. Mechanistically, ADAMTSL5 abrogation led to reduction of several oncogenic inputs relevant to $\mathrm{HCC}$, including reduced expression and/or phosphorylation levels of receptor tyrosine kinases MET, EGFR, PDGFR $\beta$, IGF1R $\beta$, or FGFR4. This phenotype was associated with significantly increased sensitivity of HCC cells to clinically relevant drugs, namely Sorafenib, Lenvatinib, Regorafenib. Moreover, ADAMTSL5 depletion drastically increased expression of AXL, accompanied by a sensitization to Bemcentinib. 
Conclusions: Our results point to a role for ADAMTSL5 in maintaining the function of key oncogenic signalling pathways, suggesting that it may act as a master regulator of tumorigenicity and drug resistance in HCC.

\section{Lay Summary}

The environment of cancer cells has profound effects on establishment, progression, and response of a tumour to treatment. Herein, we show that ADAMTSL5, a protein secreted by liver cancer cells and overlooked in cancer so far, is increased in this tumour type, is necessary for tumour formation and supports drug resistance. Adamtsl5 removal conferred sensitivity of liver cancer cells to drugs used in current treatment. This suggests ADAMTSL5 as a potential marker in liver cancer as well as a possible drug target.

\section{Highlights}

- ADAMTSL5 overexpression in HCC is associated with gene body CGI hypermethylation

- ADAMTSL5 is strongly expressed in a large fraction of human HCC

- $\quad$ Targeting ADAMTSL5 diminishes RTK inputs and interferes with tumorigenicity

- ADAMTSL5 confers tumorigenicity to sensitized, non-transformed liver cells

- Targeting ADAMTSL5 sensitizes HCC cells to drugs currently used in the clinic 


\section{Introduction}

Hepatocellular carcinoma (HCC) is the fourth-most common cause of cancer-related mortality worldwide and is growing in incidence.[1-3] It is characterized by a remarkable molecular heterogeneity among patients, with distinct (epi)genetic modifications and gene expression profile alterations.[4-6] Globally, 78 percent of liver cancer cases are secondary to chronic hepatitis B or C infections, most frequently associated with cirrhosis, a chronic disease in which fibrosis distorts liver architecture and predisposes to HCC.[6] In addition, HCC can be a sequel of alcoholic liver disease or non-alcoholic fatty liver disease (NAFLD), which are predisposing conditions whose incidence is also growing. While the knowledge of risk factors will improve likelihood of early diagnosis, current diagnostic methods show limited sensitivity and specificity, and most patients diagnosed with HCC are already at an advanced stage.[7] The perils of late diagnosis are compounded by the limited efficacy of current HCC therapies in patients with advanced-stage disease.[4, 8, 9] Sorafenib and Lenvatinib, which are first-line drugs, and second-line drugs Regorafenib, Cabozantinib, and Ramucirumab are the only approved drugs,[10] and they only marginally improve median survival . Moreover, there exist no a priori indicators of expected patient response to these treatments. Thus, there is a clear unmet need for identification of new pathways that could be targeted for therapy and for reliable biomarkers that might enable detection of early stage HCC. As in most cancers, the tumour microenvironment has a key role in HCC establishment, progression, and response to therapy.[6] Several microenvironment components affect composition and assembly of extracellular matrix (ECM), cancer cell interactions with ECM, components of the immune system, and activity of tumour-associated fibroblasts. Among these are secreted and cellsurface proteases, and several secreted molecules that associate with and modify the ECM, called matricellular proteins.[11] 
A variety of systems (-omics) approaches have been employed for identifying new disease- 


\title{
Materials and Methods
}

\begin{abstract}
Data and materials availability. The mass spectrometry proteomics data have been deposited to the ProteomeXchange Consortium (www.proteomexchange.org) via the PRIDE partner repository with the dataset identifier PXD016634.
\end{abstract}

$A \boldsymbol{A b}-\mathbf{R 2 6}^{\text {Met }}$ mice. The generation of the $R 26^{\text {stopMet }}$ mice (international nomenclature Gt(ROSA)26Sor ${ }^{\text {tml(Actb-Met)Fmai })}$ carrying a conditional mouse-human chimeric Met transgene into the Rosa26 locus, and the genotyping procedures were previously reported.[16-18]

Human samples. After Institutional Review Board approval, a search in a clinical database was performed for human HCC cases from 2002 to 2007 (Cleveland Clinic, USA; human cohort \#5). A total of $43 \mathrm{HCC}$ cases were included. Haematoxylin and eosin stained slides from $10 \%$ formalin-fixed paraffin-embedded blocks of these cases were reviewed by a liver pathologist (D.S.A.). A representative block from the tumour was identified and unstained slides on positively charged glass slides were generated for additional stains and RNAscope in situ hybridization. For RT-qPCR analyses, 18 HCC samples from Navarra University (Spain; human cohort \#3) and 16 from Montpellier University (France; human cohort \#4) were used.

Detailed methods can be found in the Supplementary Information 


\section{Results}

\section{Adamtsl5 is hypermethylated in its gene body CGI and overexpressed in clinically relevant HCC mouse models.}

Mouse Adamtsl5 has two CpG islands (CGIs), one in the promoter and the other in the gene body region (Fig. 1A). DNA methylation analyses revealed that Adamtsl5 was consistently hypermethylated in the gene body CGI in Alb-R2 $6^{\text {Met }} \mathrm{HCC}$ compared to control livers, without a change in the DNA methylation content of the promoter CGI (Fig. 1B). This alteration was accompanied by an upregulation of Adamtsl5 mRNA, as shown by RNA-seq analyses (Fig. 1C). Remarkably, treatment of $A l b-R 26^{M e t}$ mice with a DNA-demethylating agent (Decitabine) significantly decreased Adamtsl5 expression in tumours, accompanied by a consistent reduction in the methylation levels of most CpGs within its gene body (Fig. 1D, S3A, Table S1). Adamtsl5 expression was upregulated in $A l b-R 26^{M e t}$ tumours both at early stages of liver tumorigenesis and in advanced HCC compared to controls (wild-type or Alb-R26 ${ }^{M e t}$ healthy livers), correlating with increased expression of $A f p$ and $G p c 3$ (two well-characterized HCC markers), and MKi67 (a proliferation marker; Fig. 1E). The low levels of Adamtsl5 we found in the healthy mouse livers was supported by single cell RNA-seq data from the https://tabula-muris.ds.czbiohub.org/ website,[19] in which Adamtsl5 mRNA expression was virtually absent in liver cells, similar to $A f p$ and $G p c 3$, whereas Met was expressed in a high proportion of hepatocytes (Fig. S1). Low ADAMTSL5 levels in human liver were also observed (Fig. S2).

We analysed ADAMTSL5 mRNA and protein levels in Alb-R26 $6^{M e t}$ tumours compared with control livers. In situ hybridization analysis by RNAScope revealed a consistent Adamtsl5 mRNA upregulation in Alb-R26 ${ }^{M e t} \mathrm{HCC}$ in contrast with adjacent liver tissue, where Adamtsl5 mRNA was not detected (Fig. 1F). Furthermore, immunohistochemical analysis of proximate sections showed strong ADAMTSL5 staining in all analysed Alb-R26 ${ }^{\text {Met }} \mathrm{HCC}$, whereas 
ADAMTSL5 was not detected in non-tumoral tissue (Fig. 1F). Upregulation of Adamtsl5

\section{ADAMTSL5 is hypermethylated in the gene body CGI and overexpressed in a large proportion of HCC patients.}

Whereas mouse Adamtsl5 has two CGIs (Fig. 1A), human ADAMTSL5 has four CGIs: one in the promoter and three in the gene body (Fig. 2A). Analyses using HCC expression data from The Cancer Genome Atlas (TCGA) database (human cohort \#1) revealed that ADAMTSL5 mRNA levels were upregulated in 52\% of HCC cases (193/371; $\log _{2}$ fold change>1; Fig. 2B). We also examined the methylation status of ADAMTSL5 in the subgroup of HCC patients for which both methylation and expression data were available (41/371 patients). Strikingly, $86 \%$ (18/21) of patients with ADAMTSL5 overexpression displayed hypermethylation in one of the gene body CGIs (Fig. 2C, Table S2). The incidence of ADAMTSL5 CGI gene body methylation in HCC patients was further explored in a second cohort of 214 human samples (human cohort \#2: GSE56588[20]). 55\% (112/204) of these patients shared hypermethylated ADAMTSL5 gene body CGIs (Fig. S4A, Table S3). Importantly, HCC patients with high ADAMTSL5 levels were characterised by a shorter overall survival and of disease-free interval (Fig. 2D-E).

We found that upregulation of ADAMTSL5 mRNA levels correlated with alcohol intake rather than with other risk factors such as hepatitis C, hepatitis B, or NAFLD (Fig. 2F, S4B). 
Intriguingly, we found Adamtsl5 upregulation also in mice following alcohol consumption (Fig.

S4C), strengthening a possible correlation between ADAMTSL5 expression and alcohol intake observed in human patients. Interestingly, by searching correlations between ADAMTSL5 expression levels and genetic alterations in HCC patients, we observed an inverse correlation between ADAMTSL5 expression and the number of mutated genes or deletions/amplifications (Fig. 2G-H). Additionally, we found a significant difference in altered genes in the group of patients with ADAMTSL5 overexpression compared to the other two groups (Fig. 2I-J, S5, Table S4-5). Intriguingly, Dchs 1 is a gene predominantly mutated in ADAMTSL5 overexpressing patients (Fig. 2I, S5). No correlations were found with a series of clinical parameters that were analysed (Fig. S6-8). We also examined whether high ADAMTSL5 expression correlated with changes of specific markers generally used to classify HCC patients[5], which were previously used to characterise the Alb-R2 $6^{\text {Met }} \mathrm{HCC}$ model.[12] No significant differences were found among the three HCC subgroups (Fig. S9A, Table S6). Although Adamtsl5 mRNA was upregulated in the $A l b-R 26^{M e t} \mathrm{HCC}$ model, which is characterized by slightly enhanced MET levels, we did not find a correlation between ADAMTSL5 and MET levels in the cohort of HCC patients (Fig. S9B). Collectively, these results indicate that high ADAMTSL5 levels distinguish patients with specific mutations and with a predominant genetic stability, and may thus independently identify a distinct HCC patient subgroup not already classified by other HCC markers.

Next, we experimentally assessed ADAMTSL5 mRNA levels in human HCC and control specimens using samples from two independent cohorts (human cohort \#3 and \#4). RT-qPCR analysis revealed upregulation of ADAMTSL5 mRNA in 50\% (9/18) and 44\% (7/16) HCCs compared to adjacent non-tumorous livers (Fig. 3A-B, Table S7), similar to the HCC cohort from TCGA. Collectively, these findings show that ADAMTSL5 is overexpressed in a large proportion of HCC patients from independent human cohorts. Immunohistochemical analysis 
revealed ADAMTSL5 protein staining at moderate to high levels in sections from 30/43 analysed HCC patients, compared to adjacent non-tumour tissue (human cohort \#5; Fig. 3C, Table S8). In HCC and adjacent livers, staining for ADAMTSL5 and fibrillin-1 (Fig. S10A-C), a protein previously associated with liver fibrosis, were mutually exclusive.[21] Although nontumour regions of the liver were consistently ADAMTSL5-negative (Fig. 3C), weak staining was observed in some cirrhotic areas. The diverse staining patterns observed in ADAMTSL5positive tumours included weak, moderate, or intense staining of tumour cells, intense staining of macrophages bordering necrotic areas and of capillary plexuses in some tumours, and weak staining in vascular smooth muscle cells (Fig. 3D, S10D). RNAscope in situ hybridization revealed ADAMTSL5 mRNA expression by tumour cells but not by macrophages, contrasting with the immunostaining data (Fig. 3D). These observations suggest that ADAMTSL5 protein is secreted by tumour cells in a significant proportion of analysed HCC tumours, and undergoes subsequent uptake by macrophages.

\section{ADAMTSL5 is required for tumorigenic properties of $\mathrm{HCC}$ cells.}

We generated $A l b-R 26^{\text {Met }}$ HCC cell lines from distinct tumours[12] (Fig. 4A), which reproducibly showed high mRNA and protein levels of ADAMTSL5 (Fig. 4B, S11A). Consistent with reported secretion by cells,[15] ADAMTSL5 was detected in the conditioned medium of $A l b-R 26^{M e t} \mathrm{HCC}$ cells (Fig. 4B). The consistent expression in Alb-R2 $6^{M e t} \mathrm{HCC}$ cells prompted us to explore whether ADAMTSL5 influences HCC cell tumorigenic properties. Adamtsl5 expression was reduced by shRNA targeting sequences in Alb-R26 ${ }^{M e t} \mathrm{HCC}$ cells (Fig. 4C, S11B-E), which led to a striking switch in cell morphology from epithelial-like to fibroblast-like, and loss of ZO-1 and E-Cadherin expression (Fig. 4D; S11F-H).

We assessed whether the fibroblast-like shape acquired by HCC cells following Adamtsl5 targeting corresponded to an epithelial-mesenchymal transition (EMT) or to the acquisition of a "fibroblast-like" identity, by following the expression levels of specific markers through RT- 
qPCR. HCC ${ }^{\text {shAdamts15 }}$ cells, while losing Cdhl (E-cadherin) expression, gained only the expression of one mesenchymal marker, Snail, but not of Cdh2 (N-Cadherin), Vim (Vimentin), Slug, Zeb1, Zeb2, and Twist1 (Fig. 5A, S12A-S13). Concerning the TGF $\beta$ pathway, beside a slight increase in the expression of Tgfbl and Tgif2 (and Snail), we observed similar levels of Smad2 and Mtss1 (beside Twist1, Vim, CD44; Fig. 5B, S12B-S13) and a similar response to TGF $\beta 1$ stimulation in HCC shAdamts15 and control cells (Fig. S12G). Interestingly, HCC $^{\text {shAdamtsl5 }}$ cells acquired expression of fibroblast markers, such as $\alpha$-Sma, Fspl and Fbnl (Fibrillin-1; Fig. 5C, S12C-S13). We further explored the fibroblast-like identity and found that HCC ${ }^{\text {shAdamts15 }}$ cells acquired expression of Col3al, Colla1, Pcolce, which are markers of scar-associated mesenchymal cells (SAMes), while losing Krt19 expression (Fig. 5E, S12E-S13). Furthermore, $\mathrm{HCC}^{\text {shAdamts15 }}$ cells lost expression of Afp (HCC marker) as well as of Proml, Epcam, and Cdh1 (considered as cancer stem cell markers; Fig. 5D-F, S12D-F-S13). Collectively, these results indicate that loss of Adamtsl5 expression in HCC cells confers molecular/morphological fibroblast-like characteristics rather than triggering a bona fide EMT.

Functionally, we found that Adamtsl5 downregulation interfered with the ability of $A l b-R 26^{\text {Met }}$ HCC cells to form colonies in anchorage-independent assays (Fig. 6A-B, S14A) and tumour spheroids upon growth in self-renewal conditions (Fig. 6C), yet without major changes in cell

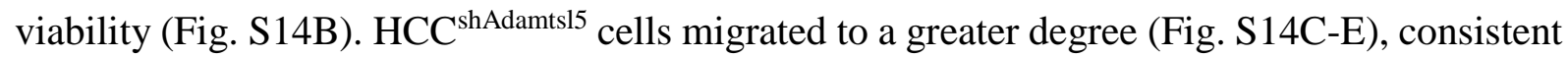
with the acquired fibroblast-like morphology (Fig. 4D), although they were not as invasive as

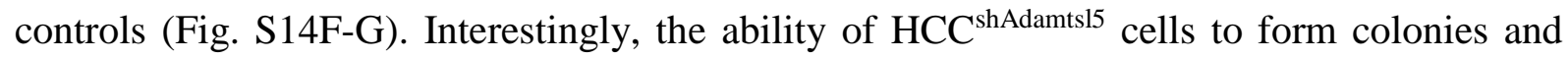
tumour spheroids, but not their morphological change, was significantly restored when they were exposed to conditioned medium from HCC cells overexpressing human ADAMTSL5 (Fig. 6D, S15).

Next, we assessed ADAMTSL5 expression and requirement in human HCC cell lines. Analysis of RNA-seq data from a panel of human HCC cells[22] revealed consistently high ADAMTSL5 
levels in all cell lines (Fig. 6E). RT-qPCR and western blot studies performed on a subset of

\section{ADAMTSL5 is required for tumorigenic properties of $\mathrm{HCC}$ cells in vivo and its overexpression is sufficient to transform sensitized hepatocytes.}

Next, we investigated whether ADAMTSL5 expression is a requirement for HCC cells tumorigenicity in vivo using xenografts. Alb-R2 $6^{\text {Met }} \mathrm{HCC}$ and $A l b-R 26^{\text {Met }} \mathrm{HCC}^{\text {shAdamts15 }}$ cells were injected subcutaneously into the flank of nude mice and tumour formation was followed over time. Compared to controls, $\mathrm{HCC}^{\text {shAdamtsl5 }}$ cells showed a striking reduction in tumour size and the percentage of animals that developed tumours (Fig. 7A-C). Additionally, we assessed the requirement for ADAMTSL5 in an experimental setting that recapitulated the liver tumour environment by performing orthotopic injections of $A l b-R 26^{M e t} \mathrm{HCC}^{\text {shControl }}$ and $\mathrm{HCC}^{\text {shAdamtsl5 }}$ cells intra-hepatically in mice. Remarkably, in contrast to controls, $\mathrm{HCC}^{\text {shAdamts15 }}$ cells did not form tumours (Fig. 7D, E), consistent with reduced in vitro tumorigenicity, loss of Afp and cancer stem cell marker expression (Fig. 5-6).

Next, we asked whether ADAMTSL5 overexpression would confer tumorigenicity to cells otherwise incapable of forming tumours. For this purpose, we chose a cellular context in which enhanced wild-type MET RTK levels, although not sufficient on their own, provide a sensitized genetic background for testing cooperativity with another pro-tumorigenic hit (Fig. 7F).[23] Whereas immorto-R26 $6^{\text {Met }}$ hepatocytes did not form tumours in immuno-compromised mice, 
human ADAMTSL5 overexpression conferred in vivo tumorigenicity to these cells (Fig. 7G-I).

Collectively, these in vivo studies underline the oncogenic properties of ADAMTSL5 in HCC.

\section{ADAMTSL5 expression correlates with high dosage of several oncogenes relevant to HCC.}

We performed mass spectrometry for unbiased comparison of the secretome of $\mathrm{HCC}^{\text {shControl }}$ and $\mathrm{HCC}^{\text {shAdamts15 }}$ cells. Excluding ribosome-related proteins frequently detected in such analyses, we found 614 proteins with differential abundance in the two secretomes: 348 upregulated and

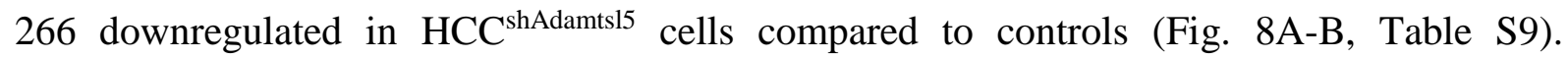
According to the Kyoto Encyclopedia of Genes and Genomes (KEGG) database, several cancer-related pathways were significantly enriched, such as axon guidance (frequently overrepresented in cancer), ECM-receptor interaction and adhesion components, proteoglycans and glycans (Fig. 8C, Table S9). These pathways appear to be consistent with ADAMTSL5 effects on cell morphology and tumorigenicity, and indicated that loss of tumorigenicity following Adamtsl5 knock-down was accompanied by depletion of several oncogenic signals. We selected some proteins for biochemical assessment in the secretomes and cell lysates of $\mathrm{HCC}^{\text {shControl }}$ and $\mathrm{HCC}^{\text {shAdamts15 }}$ cells (Fig. 8D, S17A). We found a striking reduction of the RTK MET extracellular domain in the medium and of full-length MET in HCC $\mathrm{HAdamts15}^{\text {cell lysates }}$ (Fig. 7D-E, S17E). Protein levels of EPHA2 and EPHA4, two other RTKs also implicated in HCC, $[24,25]$ were reduced (although levels of a smaller band detected with an EPHA4 antibody in cell lysates were increased; Fig. 8D-E, S17E). Additionally, UNC5B (a netrinreceptor, which is a prognostic marker of HCC; Fig. S17B) and LPHN3 (Latrophilin3, which interacts with UNC5B and regulates cell adhesion[26]), each a trans-membrane protein, were less abundant in the secretome of $\mathrm{HCC}^{\text {shAdamts15 }}$ cells, but remained unchanged in cell lysates (Fig. 8D-E). Furthermore, we found a reduction of cleaved products of FAT1 (which acts as an oncogene in $\mathrm{HCC}[27])$ in the medium, likely resulting from extracellular shedding [28] (Fig. 
S17C-D). We also detected an upregulation of biglycan (a small leucine-rich repeat

\section{ADAMTSL5 depletion sensitizes HCC cells to drugs used for HCC treatment in the clinic.}

The severe depletion of several RTKs relevant to tumorigenicity and resistance of HCC cells to drugs used for HCC treatment in the clinic prompted us to explore whether ADAMTSL5 downregulation influenced $\mathrm{HCC}$ cell sensitivity to these drugs. The Alb-R2 $6^{\mathrm{Met}} \mathrm{HCC}$ cellular system was particularly appropriate for addressing this question because of its resistance to 
Sorafenib, which was previously reported.[12] We compared the sensitivity of HCC shAdamts15 $^{\text {she }}$ and control cells to several multi-kinase inhibitors: Crizotinib, Lenvatinib, Regorafenib, and Sorafenib. Because of the striking upregulation of AXL observed in Adamtsl5-downregulated cells, we also assessed the effects of Bemcentinib, an inhibitor of AXL (Table S10). HCC shControl $^{\text {s. }}$ cells were either totally resistant (Lenvatinib) or displayed limited sensitivity when drugs were used at high dosage (Fig. 8I). In contrast, $\mathrm{HCC}^{\text {shAdamts } 15}$ cell viability was significantly decreased when cells were exposed to Crizotinib, Lenvatinib, Regorafenib, and to a lesser extent, to Sorafenib (Fig. 8I). Remarkably, Bemcentinib treatment drastically interfered with HCC $^{\text {shAdamts15 }}$ cell viability whereas no effect was observed in control cells (Fig. 8I). Overall, our results illustrate that ADAMTSL5 targeting in HCC cells leads to a profound alteration of several oncogenic inputs such as those linked to RTKs, and confers sensitivity to clinically relevant drugs. 


\section{Discussion}

Lack of efficient therapies for HCC provides a strong incentive for seeking new disease pathways and more reliable biomarkers, notably those that can be used for diagnosis of early stages of HCC. Biomarkers for early detection and patient stratification will be even more important in the future, when additional first- and second-line therapies for liver cancer may become available. Here, we identified ADAMTSL5 upregulation in a large percentage of HCC patients from several independent cohorts: according to RNA-seq data or our RT-qPCR analyses, ranging from 44 to $52 \%$ of human HCC patients; according to immunohistochemistry analysis, $70 \%$ of $\mathrm{HCC}$ patients. Adamtsl 5 upregulation was reproduced in the $A l b-R 26^{M e t} \mathrm{HCC}$ model and importantly, its increased expression was already evident during early liver tumorigenesis. Adamtsl5 upregulation was similarly detected in $N-R_{a s}^{G 12 D}: p 53^{\text {null }}$ mouse liver tumours, indicating that its overexpression is not restricted to a single genetic trigger during mouse tumorigenesis. We have shown, both in mouse and human tumours, that augmented ADAMTSL5 expression correlates with hypermethylation of its gene body CGI, an epigenetic mechanism that we recently reported to be involved in the regulation of oncogene expression.[13] Thus, our data suggest that hypermethylation of gene body CGI is predictive, although not exclusively, of high ADAMTSL5 expression in HCC. While these data require further validation in large patient cohorts, they imply a potential clinical significance of ADAMTSL5 upregulation in a subgroup of patients and might qualify ADAMTSL5 as a potential HCC biomarker. Interestingly, in situ analysis indicated that ADAMTSL5 was produced by $\mathrm{HCC}$ cells in the $A l b-R 26^{M e t} \mathrm{HCC}$ model, as well as in roughly half of human HCC cases. In agreement with the described heterogeneity of HCCs and with mRNA expression observed in about half of the cases annotated by TCGA, we found that a significant proportion of human HCC samples stained negative for ADAMTSL5. However, one caveat of analysing biopsies, either for RNA content or by staining, is that of sampling variability, i.e. samples may 
come from ADAMTSL5-negative areas of heterogeneous, often multifocal tumours that may contain expressing cells elsewhere. Therefore, it is noteworthy that as a secreted protein, ADAMTSL5 is a potential candidate biomarker that is accessible through liquid biopsy. Indeed, liquid biopsy can overcome limitations of tissue biopsy, since it is expected to represent the entire molecular picture of a patient's malignancy. Future studies will determine if measuring plasma ADAMTSL5 levels and/or hypermethylation of the gene body ADAMTSL5 CGI in circulating cell-free DNA could be useful as a HCC biomarker. It will also be important to ascertain if human HCC can be stratified by ADAMTSL5 status to uncover histological, precancerous or risk associations, which may contribute to a better-informed choice of therapeutic options.

We illustrated by a series of in vitro and in vivo assays that increased levels of ADAMTSL5 are associated with tumorigenic properties of HCC cells. Indeed, ADAMTSL5 targeting interfered with HCC cell anchorage-dependent and -independent growth, tumour spheroid formation and with orthotopic and subcutaneous xenograft growth. The oncogenic function of ADAMTSL5 is further supported by our data showing that its overexpression conferred tumorigenicity to sensitized, non-transformed liver cells. Collectively, these results indicate that ADAMTSL5, which is produced by HCC cells, may be a key factor determining their tumorigenicity. Of note, the secreted ADAMTSL5 acts in a non-cell autonomous fashion, as demonstrated by the partial restoration of the phenotype of $\mathrm{HCC}^{\text {shAdamtsl5 }}$ cells by the conditioned medium of cells over-expressing ADAMTSL5. Importantly, since the secretome of ADAMTSL5-expressing cells is profoundly altered, it is thus possible that some of its oncogenic effects are indirect.

Interestingly, increased ADAMTSL5 methylation was recently reported in chemotherapyresistant acute lymphoblastic leukaemia patients.[32] However, neither the genomic location of such hypermethylation nor the consequences for ADAMTSL5 expression in acute lymphoblastic 
leukaemia patients have been investigated. In addition, recent studies implicated ADAMTSL5 in pathologies other than cancer.[33]

In summary, we have shown that ADAMTSL5, which is overexpressed in a significant proportion of HCC patients, has an essential role in HCC pathogenesis. Our results suggest that ADAMTSL5 is a potential master regulator of HCC, acting upstream of several key oncogenic pathways, including RTKs, such as MET, EGFR, GAB1, PDGFR $\beta$, IGF1R $\beta$, and FGFR4. Intriguingly, ADAMTSL5 targeting results in decreased activation of several RTK signals, irrespectively on changes of their protein levels. Strikingly, ADAMTSL5 depletion gives rise to a dramatic increase of AXL. It is tempting to speculate that upregulation of AXL may occur in response to loss of several other RTK inputs. This interpretation is supported by the acquired vulnerability of $\mathrm{HCC}^{\text {shADAMTSL5 }}$ to AXL inhibition, suggesting a novel therapeutic strategy for a defined subset of HCCs. From a general perspective, a number of RTK-targeting drugs are now available and our results suggest that some could be repurposed for treating ADAMTSL5overexpressing HCC, especially when used in combination with agents targeting ADAMTSL5, which remain to be developed. 


\section{Acknowledgments}

These results are in part based upon public data generated by TCGA Research Network: http://cancergenome.nih.gov/. We thank: all members of our labs for helpful discussions and comments; A. Porras and F. Helmbacher for extremely valuable feedback on the study; B. Habermann and A. Yim for assisting us on evaluation of human TCGA datasets; Á.M. Martínez-Valverde for providing us the SV40 Large T Antigen to immortalize the embryonic hepatocytes; S. Lev for advises to produce lentivirus particles carrying shRNA and for extremely valuable feedback; C. Berasain, M. Elizalde, and I. Uriarte for providing us samples from human cohort \#3 and from mice following alcohol consumption; people at the IBDM mouse facility for excellent help with mouse husbandry; A. Dobric and C. Giaccherini for their contributions to studies on molecular and functional characterizations of Adamtsl5 shRNAtargeted HCC cells; D. Reinhardt for anti-fibrillin-1 antibody. 


\section{References}

[1] McGlynn KA, Petrick JL, El-Serag HB. Epidemiology of Hepatocellular Carcinoma. Hepatology 2020.

[2] Yang JD, Hainaut P, Gores GJ, Amadou A, Plymoth A, Roberts LR. A global view of hepatocellular carcinoma: trends, risk, prevention and management. Nat Rev Gastroenterol Hepatol 2019;16:589-604.

[3] Villanueva A. Hepatocellular Carcinoma. The New England journal of medicine 2019;380:1450-1462.

[4] Llovet JM, Villanueva A, Lachenmayer A, Finn RS. Advances in targeted therapies for hepatocellular carcinoma in the genomic era. Nat Rev Clin Oncol 2015;12:408-424.

[5] Zucman-Rossi J, Villanueva A, Nault JC, Llovet JM. Genetic Landscape and Biomarkers of Hepatocellular Carcinoma. Gastroenterology 2015;149:1226-1239 e1224.

[6] Llovet JM, Zucman-Rossi J, Pikarsky E, Sangro B, Schwartz M, Sherman M, et al. Hepatocellular carcinoma. Nat Rev Dis Primers 2016;2:16018.

[7] Sherman M, Bruix J, Porayko M, Tran T, Committee APG. Screening for hepatocellular carcinoma: the rationale for the American Association for the Study of Liver Diseases recommendations. Hepatology 2012;56:793-796.

[8] Llovet JM, Hernandez-Gea V. Hepatocellular Carcinoma: Reasons for Phase III Failure and Novel Perspectives on Trial Design. Clinical Cancer Research 2014;20:2072-2079.

[9] Sprinzl MF, Galle PR. Current progress in immunotherapy of hepatocellular carcinoma. J Hepatol 2017;66:482-484.

[10] Montironi C, Montal R, Llovet JM. New Drugs Effective in the Systemic Treatment of Hepatocellular Carcinoma. Clin Liver Dis (Hoboken) 2019;14:56-61. 
[11] Gerarduzzi C, Hartmann U, Leask A, Drobetsky E. The Matrix Revolution: Matricellular Proteins and Restructuring of the Cancer Microenvironment. Cancer Res 2020;80:2705-2717.

[12] Fan YN, Arechederra M, Richelme S, Daian F, Novello C, Calderaro J, et al. A Phosphokinome-Based Screen Uncovers New Drug Synergies for Cancer Driven by LiverSpecific Gain of Nononcogenic Receptor Tyrosine Kinases. Hepatology 2017;66:1644-1661.

[13] Arechederra M, Daian F, Yim A, Bazai SK, Richelme S, Dono R, et al. Hypermethylation of gene body $\mathrm{CpG}$ islands predicts high dosage of functional oncogenes in liver cancer. Nat Commun 2018;9:3164.

[14] Cassol F, Portal L, Richelme S, Dupont M, Boursier Y, Arechederra M, et al. Tracking Dynamics of Spontaneous Tumors in Mice Using Photon-Counting Computed Tomography. iScience 2019;21:68-83.

[15] Bader HL, Wang LW, Ho JC, Tran T, Holden P, Fitzgerald J, et al. A disintegrin-like and metalloprotease domain containing thrombospondin type 1 motif-like 5 (ADAMTSL5) is a novel fibrillin-1-, fibrillin-2-, and heparin-binding member of the ADAMTS superfamily containing a netrin-like module. Matrix Biol 2012;31:398-411.

[16] Tonges L, Ostendorf T, Lamballe F, Genestine M, Dono R, Koch JC, et al. Hepatocyte growth factor protects retinal ganglion cells by increasing neuronal survival and axonal regeneration in vitro and in vivo. J Neurochem 2011;117:892-903.

[17] Genestine M, Caricati E, Fico A, Richelme S, Hassani H, Sunyach C, et al. Enhanced neuronal Met signalling levels in ALS mice delay disease onset. Cell Death Dis 2011;2:e130.

[18] Fan Y, Richelme S, Avazeri E, Audebert S, Helmbacher F, Dono R, et al. TissueSpecific Gain of RTK Signalling Uncovers Selective Cell Vulnerability during Embryogenesis. PLoS genetics 2015;11:e1005533. 
[19] Tabula Muris C, Overall c, Logistical c, Organ c, processing, Library p, et al. Singlecell transcriptomics of 20 mouse organs creates a Tabula Muris. Nature 2018;562:367-372.

[20] Villanueva A, Portela A, Sayols S, Battiston C, Hoshida Y, Mendez-Gonzalez J, et al. DNA methylation-based prognosis and epidrivers in hepatocellular carcinoma. Hepatology $2015 ; 61: 1945-1956$.

[21] Lorena D, Darby IA, Reinhardt DP, Sapin V, Rosenbaum J, Desmouliere A. Fibrillin-1 expression in normal and fibrotic rat liver and in cultured hepatic fibroblastic cells: modulation by mechanical stress and role in cell adhesion. Lab Invest 2004;84:203-212.

[22] Caruso S, Calatayud AL, Pilet J, La Bella T, Rekik S, Imbeaud S, et al. Analysis of Liver Cancer Cell Lines Identifies Agents With Likely Efficacy Against Hepatocellular Carcinoma and Markers of Response. Gastroenterology 2019;157:760-776.

[23] Fan YN, Bazai SK, Daian F, Arechederra M, Richelme S, Temiz NA, et al. Evaluating the landscape of gene cooperativity with receptor tyrosine kinases in liver tumorigenesis using transposon-mediated mutagenesis. Journal of Hepatology 2019;70:470-482.

[24] Yan Y, Luo YC, Wan HY, Wang J, Zhang PP, Liu M, et al. MicroRNA-10a Is Involved in the Metastatic Process by Regulating Eph Tyrosine Kinase Receptor A4-Mediated EpithelialMesenchymal Transition and Adhesion in Hepatoma Cells. Hepatology 2013;57:667-677.

[25] Jin RA, Lin H, Li GH, Xu JJ, Shi L, Chang C, et al. TR4 nuclear receptor suppresses HCC cell invasion via downregulating the EphA2 expression. Cell Death \& Disease 2018;9.

[26] Lu YC, Nazarko OV, Sando R, 3rd, Salzman GS, Li NS, Sudhof TC, et al. Structural Basis of Latrophilin-FLRT-UNC5 Interaction in Cell Adhesion. Structure 2015;23:1678-1691. [27] Valletta D, Czech B, Spruss T, Ikenberg K, Wild P, Hartmann A, et al. Regulation and function of the atypical cadherin FAT1 in hepatocellular carcinoma. Carcinogenesis 2014;35:1407-1415. 
[28] Caruso N, Herberth B, Bartoli M, Puppo F, Dumonceaux J, Zimmermann A, et al. Deregulation of the protocadherin gene FAT1 alters muscle shapes: implications for the pathogenesis of facioscapulohumeral dystrophy. PLoS genetics 2013;9:e1003550.

[29] Niu ZS, Niu XJ, Wang WH. Role of the receptor tyrosine kinase Axl in hepatocellular carcinoma and its clinical relevance. Future Oncol 2019;15:653-662.

[30] Pinato DJ, Brown MW, Trousil S, Aboagye EO, Beaumont J, Zhang H, et al. Integrated analysis of multiple receptor tyrosine kinases identifies Axl as a therapeutic target and mediator of resistance to sorafenib in hepatocellular carcinoma. Brit J Cancer 2019;120:512-521.

[31] Raja A, Park I, Haq F, Ahn SM. FGF19-FGFR4 Signaling in Hepatocellular Carcinoma. Cells 2019;8.

[32] Abdullah M, Choo CW, Alias H, Abdul Rahman EJ, Mohd Ibrahim H, Jamal R, et al. ADAMTSL5 and CDH11: putative epigenetic markers for therapeutic resistance in acute lymphoblastic leukemia. Hematology 2017;22:386-391.

[33] Bonifacio KM, Kunjravia N, Krueger JG, Fuentes-Duculan J. Cutaneous Expression of A Disintegrin-like and Metalloprotease domain containing Thrombospondin Type 1 motif-like 5 (ADAMTSL5) in Psoriasis goes beyond Melanocytes. J Pigment Disord 2016;3. 


\section{Figure Legends}

Fig. 1. Adamtsl5 is hypermethylated in the gene body CGI and overexpressed in Alb-R26 ${ }^{\text {Met }}$ tumours compared with control livers. (A) Schematic representation of the mouse Adamtsl5 locus (blue: exons; CGIs: green). (B) Methylation levels of the Adamtsl5 gene body and promoter CGIs in control livers and Alb-R26 $6^{\text {Met }}$ tumours. Note significantly higher Adamtsl5 methylation levels in gene body CGI in Alb-R26 $6^{\text {Met }}$ tumours, with no change in the promoter CGI. (C) Adamtsl5 mRNA levels in Alb-R26 $6^{\text {Met }}$ tumours versus controls. (D) Methylation levels of CpGs within the Adamtsl5 gene body CGI (left) and Adamtsl5 expression levels (right) in tumours dissected from $A l b-R 26^{M e t}$ mice untreated (red) or treated with decitabine (green). (E) mRNA expression levels of Adamtsl5, Afp, Gpc3 (two HCC markers), and Mki67 (proliferative marker). Note Adamtsl5 upregulation in Alb-R26 $6^{\text {Met }}$ early tumours (smaller than $3 \mathrm{~mm}$ ) and HCC. (F) Adamtsl5 mRNA (left) and protein (right) in Alb-R26 $6^{\text {Met }}$ tumours versus adjacent control livers. (G) Increased Adamtsl5 mRNA levels in $N-R a s^{G 12 D}: p 53^{\text {null }}$ mouse tumours versus adjacent livers. n.s.:not significant; *:P<0.05; **:P<0.01; ***:P<0.001 (Mann-Whitney: B,C,E,G,H; Kruskall-Wallis: D).

Fig. 2. ADAMTSL5 expression correlates with hypermethylated gene body CGIs and with a worse prognosis in human HCC. (A) Scheme of the human ADAMTSL5 locus (blue: exons; CGIs: green). (B) ADAMTSL5 mRNA levels in HCC patients (cohort \#1). (C) ADAMTSL5 mRNA (middle) and methylation (bottom) levels in HCC patients. Bottom: Hypermethylated CGI (black line) in the ADAMTSL5 promoter and gene body in the corresponding patients. Note hypermethylated gene body CGIs in most HCC patients with ADAMTSL5 overexpression (86\%, 18/21). (D-E) Kaplan-Meier curves reporting the probability of the overall survival (D) and disease-free interval (E) according to ADAMTSL5 levels. (F) Diagram reporting the presence (blue line) of major HCC risk factors. HCC patients with higher ADAMTSL5 mRNA levels showed a significant, although not exclusive, association with alcohol consumption. (G- 
$\mathrm{H})$ Number of mutated genes $(\mathrm{G})$ or deletions/amplifications $(\mathrm{H})$ according to ADAMTSL5 levels (cohort \#1). (I) Heatmap reporting genes mutated only in one or two groups corresponding to ADAMTSL5 levels, taking into consideration the top 10 ranked positions determined by the percentage of patients carrying mutations in the indicated genes. Red and green: upper and lower gene ranking (corresponding to high and low percentage of patients), respectively. White: absence of mutations in patients. On the right, TP53 and CTNNB1 (among the most frequently mutated genes) are also reported. (J) Heatmap reporting genes with copy number alterations in the HCC patient subgroups corresponding to ADAMTSL5 levels, taking into consideration the top 10 ranked positions determined by the percentage of patients carrying genetic alterations of the indicated genes. Detailed information about altered genes in the three groups are reported in Figure S5 and Table S4-5. Two-way ANOVA followed by Tukey multiple comparison: statistical significance between UP versus DOWN groups, DOWN versus NO CHANGE (I); between UP versus DOWN groups, UP versus NO CHANGE, DOWN versus NO CHANGE (J). P<0.0001.

Fig. 3. ADAMTSL5 upregulation and ADAMTSL5 localization in human HCC patient cohorts. (A-B) ADAMTSL5 mRNA upregulation in HCC samples versus adjacent nontumorous liver (cohort \#3: 50\%; cohort \#4: 44\%). (C) Immunohistochemical analysis revealed strong ADAMTSL5 levels in HCC regions, but not adjacent non-tumorous liver. ADAMTSL5 staining was localized to HCC cells and to macrophages in necrotic areas. (D) ADAMTSL5 protein and mRNA in proximate HCC sections. Note ADAMTSL5 RNA expression in HCC cells (arrowheads), but not in macrophages (arrows). ADAMTSL5 protein in macrophages indicates its possible uptake by these cells during clearance of necrotic areas.

Fig. 4. ADAMTSL5 is expressed in Alb-R26 $6^{M e t} \mathrm{HCC}$ cells and its downregulation leads to a switch from epithelial- to fibroblast-like characteristics. (A) Scheme of $A l b-R 26^{M e t}$ 
HCC $^{\text {shAdamts15 }}$ cell generation. (B) ADAMTSL5 protein levels in cell lysates and in conditioned medium (CM) of HCC3, HCC13, and HCC14. CM from untransfected HEK cells or HEK cells transfected with a human ADAMTSL5 (hA) was used as the control. (C) Down-regulation of ADAMTSL5 protein levels in Alb-R26 $6^{\text {Met }} \mathrm{HCC} 13^{\text {shControl }}$ (cntr) and $\mathrm{HCC} 13^{\text {shAdamts15 }}$ cells (four clones). Two different Adamtsl5 shRNAs were used (sh3 and sh1). Equal loading is shown (Ponceau). (D) Immunofluorescence showing loss of ADAMTSL5, ZO1, and E-Cadherin in Alb-R26 $6^{\text {Met }} \mathrm{HCC} 13^{\text {shAdamts15 }}$ cells versus controls. HCC13 $3^{\text {shAdamts15 }}$ cells acquired a fibroblastlike phenotype.

Fig. 5. ADAMTSL5 downregulation in HCC cells leads to a switch in the expression of several cellular markers. mRNA levels of the indicated genes in $A l b-R 26^{M e t} \mathrm{HCC}^{\text {shControl }}$ (cntr) and $\mathrm{HCC}^{\text {shAdamts15_clone3.7 }}$ (sh3.7) cells. Genes correspond to markers of epithelial/mesenchymal cells (A), TGF $\beta$ pathway (B), fibroblasts (C), HCC (D), fibroblast subtypes (E), cancer stem cells $(\mathrm{F}) . *: \mathrm{P}<0.05 ; * *: \mathrm{P}<0.01 ; * * *: \mathrm{P}<0.001$ (Student $\mathrm{t}$-test). Heatmaps report downregulated (green), upregulated (red) and unchanged (black) mRNAs, or no expression (grey). Values are reported as $\log _{2}$ fold change of expression levels in $\mathrm{HCC}^{\text {shAdamts15_clone3.7 }}$ compared to controls (complementary data: Figure S12-S13).

Fig. 6. ADAMTSL5 is required for in vitro tumorigenic properties of mouse and human

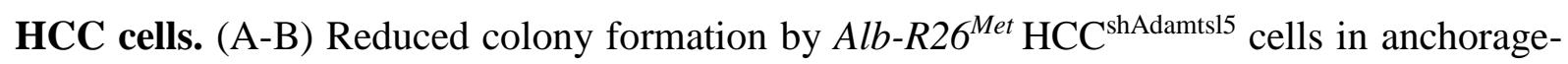
independent growth assays. Note significant restoration of tumorigenic properties after exposing $\mathrm{HCC}^{\text {shAdamts15 }}$ cells to the HCC secretome with overexpressed hADAMTSL5 $(\mathrm{CM}+\mathrm{hA}) .(\mathrm{C})$ Numbers of tumour spheroids formed by HCC13 $3^{\text {shAdamtsl5 }}$ cells and controls. (D) Rescue of tumour spheroid formation upon exposing $\mathrm{HCC} 13^{\text {shAdamtsl5 }}$ cells to a HCC secretome with hADAMTSL5 overexpression (CM+hA). (E) ADAMTSL5 mRNA levels in a panel of human HCC cells, based on data from [22]. (F) ADAMTSL5 protein levels in conditioned 
medium and cell lysates (HCC13 cells used as controls). (G-I) ADAMTSL5 downregulation in

Fig. 7. ADAMTSL5 expression confers tumorigenic properties to cells in vivo. (A-C) Images of dissected tumours (A) and quantification of mean tumour volume over time (B) and at 8 weeks $(\mathrm{C})$ in mice injected subcutaneously with $A l b-R 26^{\text {Met }} \mathrm{HCC} 13$ and $\mathrm{HCC}^{\text {shAdamts15 }}$ cells. In (C), grey dots correspond to tumours dissected earlier than 8 weeks for ethical reasons. (DE) Images of dissected livers (arrows indicate tumours) and quantification of tumour volume at 5 weeks following orthotopic injection with $A l b-R 26^{M e t} \mathrm{HCC} 13^{\text {shControl }}$ and $\mathrm{HCC}{ }^{\text {shAdamtsl5 }}$ cells. (F) Scheme reporting the establishment of immorto-R26 $6^{\text {Met }}$ sensitized hepatocytes without and with human ADAMTSL5 (h-ADAMTSL5) expression (immorto-R26 $6^{\text {Met }}$ hepa $^{\text {overADAMTSL5 }}$ ). (GI) Images of mice $(\mathrm{G})$, quantification of mean tumour volume over time $(\mathrm{H})$ and at 11 weeks (I) in mice injected subcutaneously with immorto-R26 $6^{\text {Met }}$ control hepatocytes (hepa) and immorto-R2 $6^{\text {Met }}$ hepatocytes ${ }^{\text {overADAMTSL5 }}$ (hepa $\left.{ }^{\text {overADAMTSL5}}\right)$. Arrows indicate tumours. $*: \mathrm{P}<0.05 ; * *: \mathrm{P}<0.01 ; * * *: \mathrm{P}<0.001$ (Mann-Whitney test).

Fig. 8. ADAMTSL5 downregulation leads to suppression of several oncogenic signals in HCC cells. (A-B) Volcano plot (A) and heatmap (B), reporting proteins found significantly downregulated or upregulated in the secretome of $A l b-R 26^{M e t} \mathrm{HCC}^{\text {shAdamts15_clone3.7 }}$ compared with $\mathrm{HCC}^{\text {shControl }}$ cells. Orange dots in (A) correspond to proteins biochemically validated by 
western blots. (C) Enrichment KEGG pathway for differentially abundant proteins in the

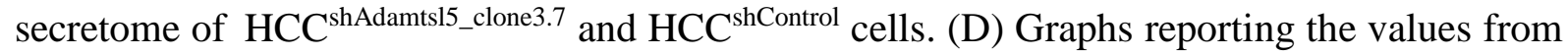
mass spectrometry analysis for the biochemically validated proteins (shown in E). 5 independent biological replicates each with 3 experimental replicates were used. Some of the proteins were undetectable because of low levels. (E) Protein levels in the secretome and cell lysates of $\mathrm{HCC}^{\text {shControl }}$ (cntr) and $\mathrm{HCC}^{\text {shAdamts15_clone3.7 }}$ (sh3.7) cells. For secretome analysis, samples were normalized to protein in cell lysates. (F) mRNA levels of the indicated genes. $(\mathrm{G})$ Protein expression and phosphorylation levels. E and G: quantifications in Table S10D. $(\mathrm{H})$ mRNA levels of the indicated genes. (I) Cell viability in the absence (NT) or in the presence of the indicated drugs. ns.: not significant; $*: \mathrm{P}<0.05 ; * *: \mathrm{P}<0.01 ; * * *: \mathrm{P}<0.001$ (student $\mathrm{t}$-test: D,F,H; two-way ANOVA: I). 
A

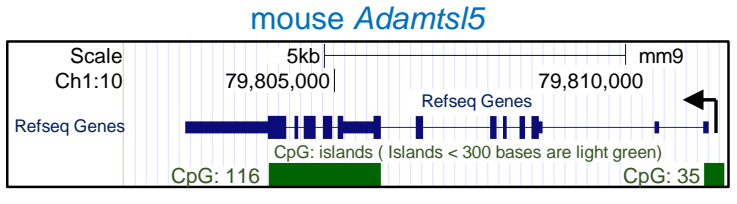

B

AdamtsI5 CGI

methylation levels

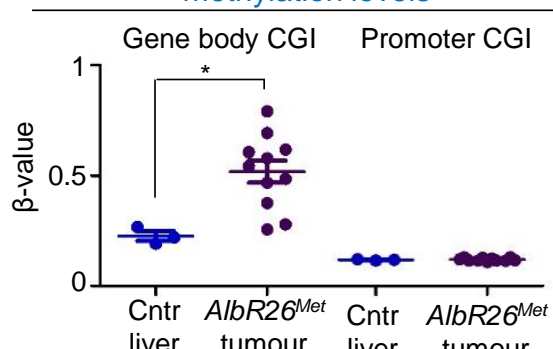

D

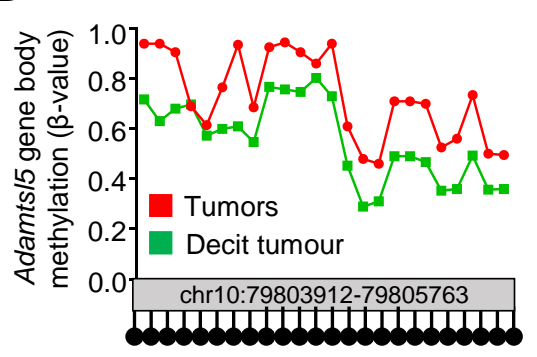

$\mathbf{E}$

$\square$ WT Liver $(\mathrm{n}=6)$

Alb-R26 ${ }^{\text {Met }}$ liver $(n=4)$
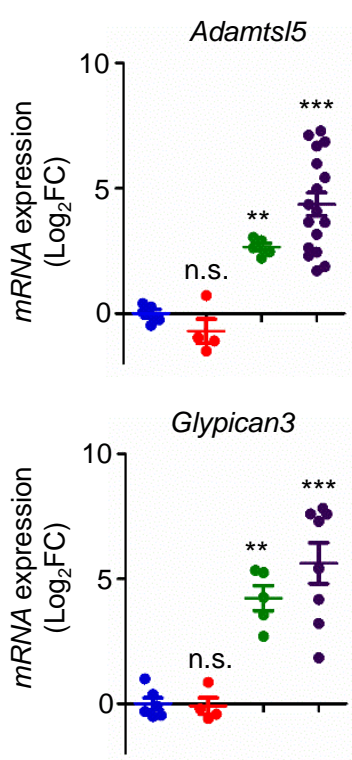

C

Adamts/5 expression levels (RNA-seq)
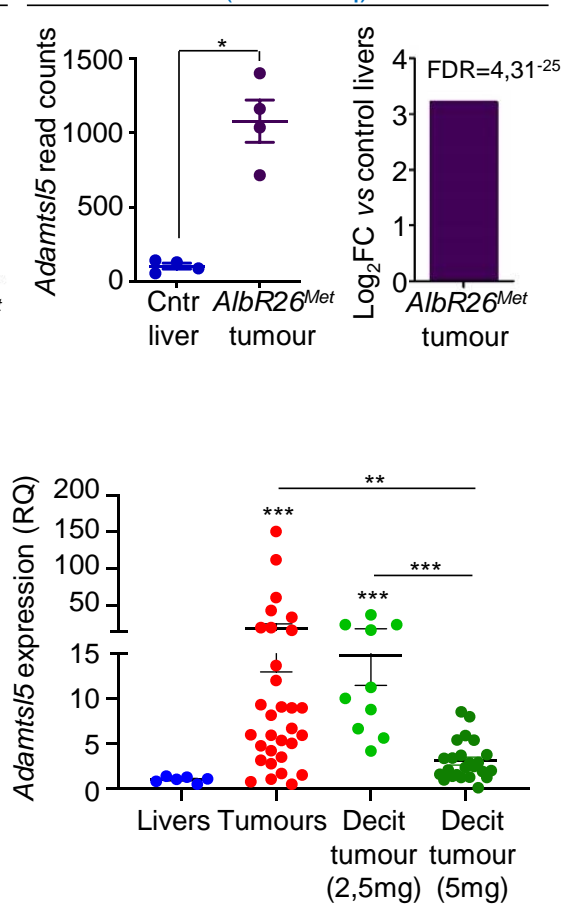

Early Alb-R26 Met tumour ( $\mathrm{n}=5)$

Advanced Alb-R26 $6^{\text {Met }}$ tumour $(\mathrm{n}=17)$
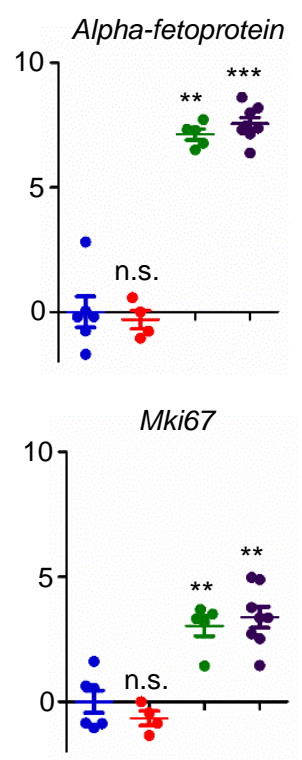

F Adamts/5 mRNA ADAMTSL5 protein

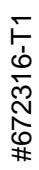

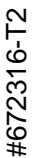

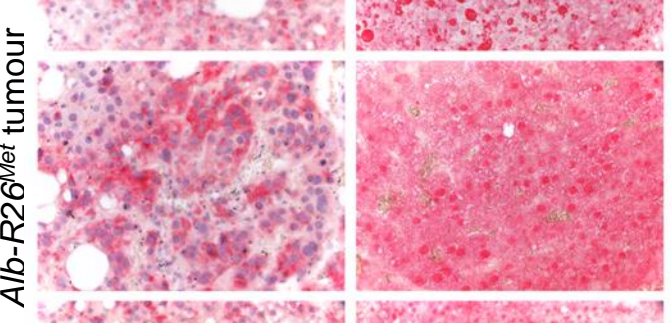

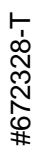

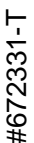

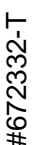

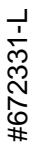

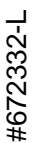

G

$N-R A S^{G 12 D}: p 53^{\text {null }}$ tumour

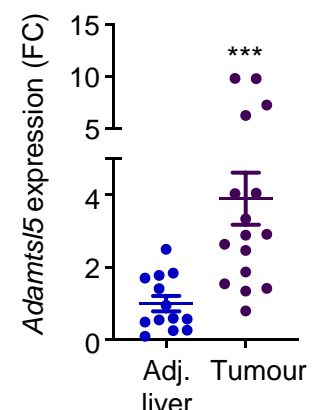

Figure 1 
A

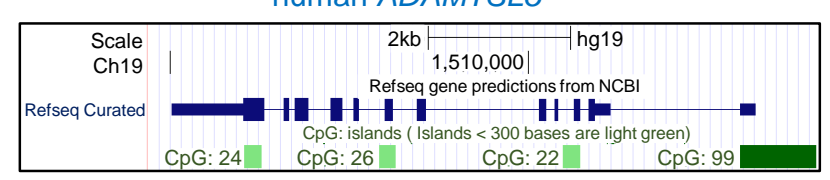

B

ADAMTSL5 expression - RNA-seq data (human cohort \#1: 371 HCC patients; TCGA)

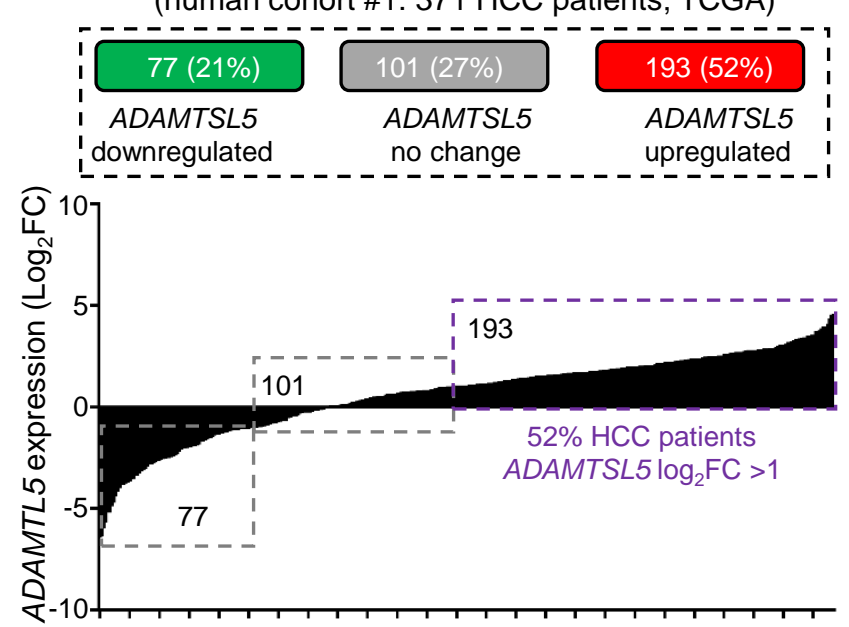

371 HCC patients (from TCGA)

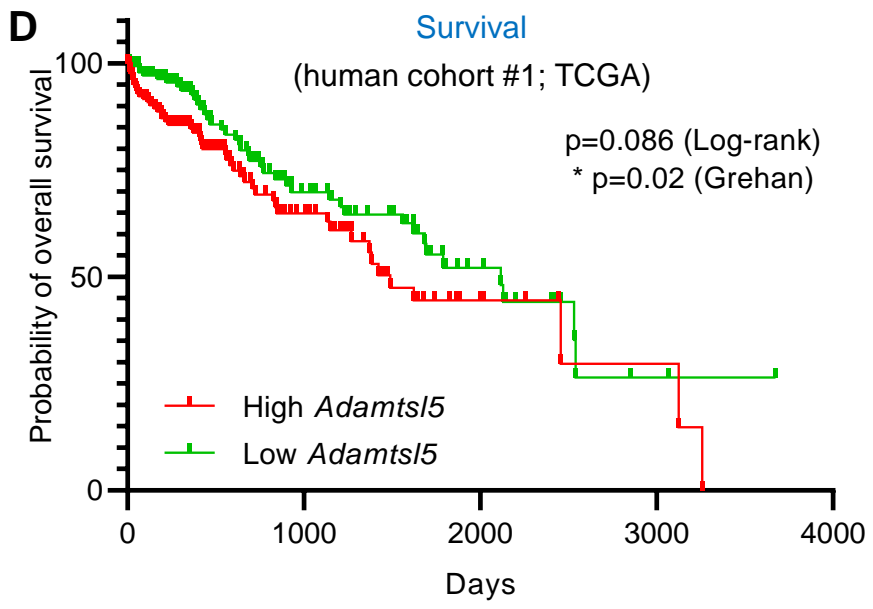

$\mathbf{F}$ ADAMTSL5

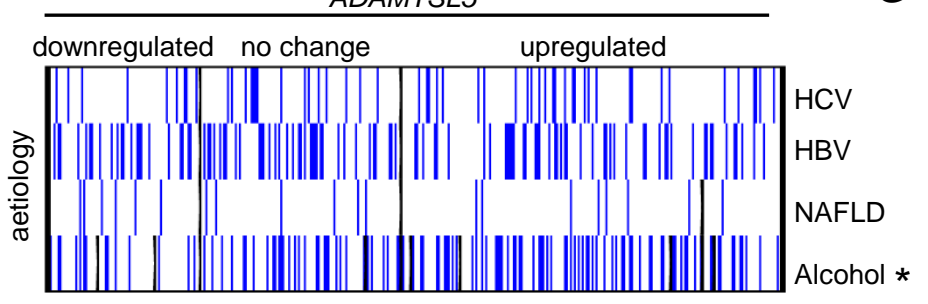

(human cohort \#1; TCGA)
C

RNA-seq and methylome data (human cohort \#1: 41 HCC patients; TCGA)
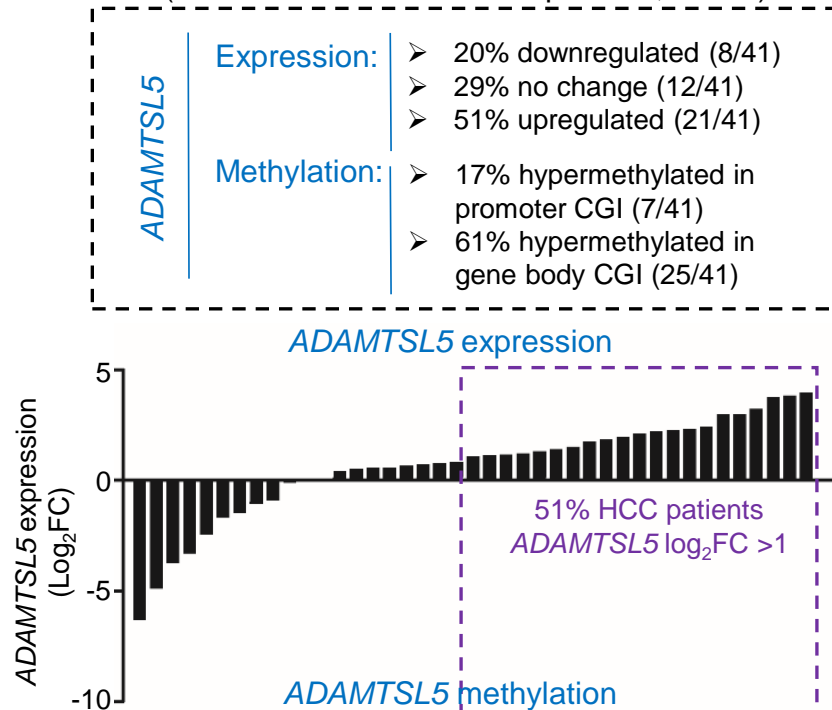

ADAMTSL5 expression
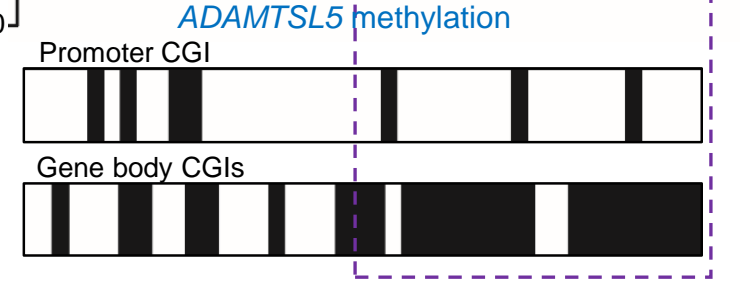

Methylation difference: $\quad \mathbf{~}>0,1 \quad \square<0,1$

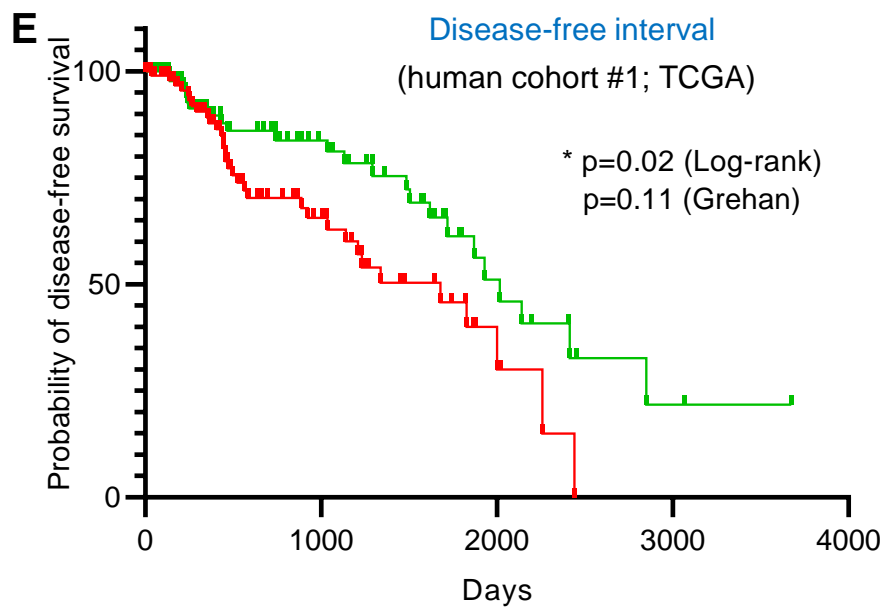

G

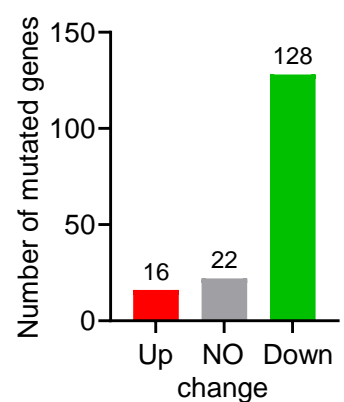

I

Genes mutated in HCC patients according to ADAMTSL5 levels

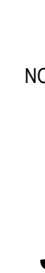

OCAN 

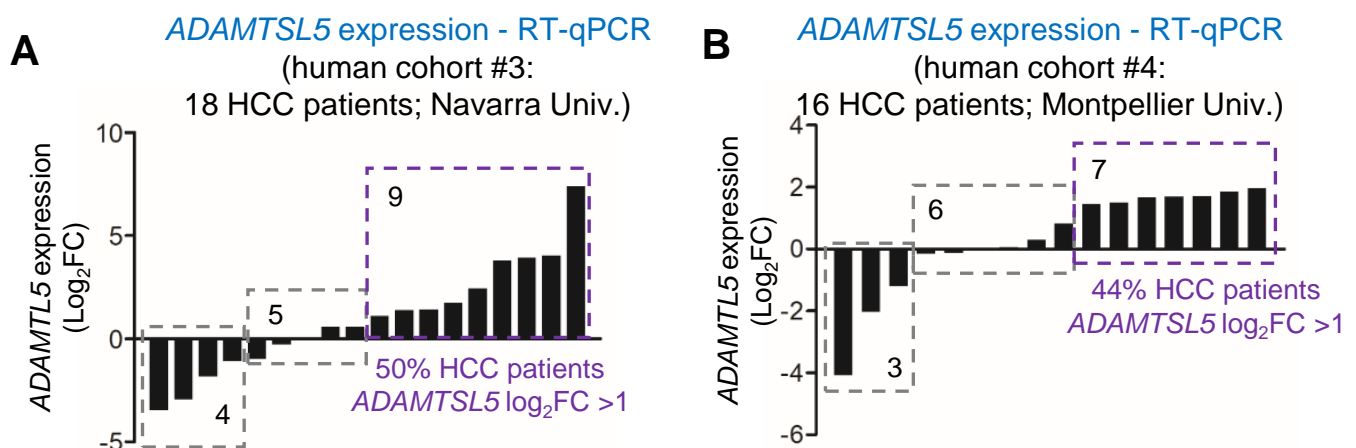

ADAMTSL5 protein
(human cohort \#5: 43 HCC patients; Cleveland Clinic)

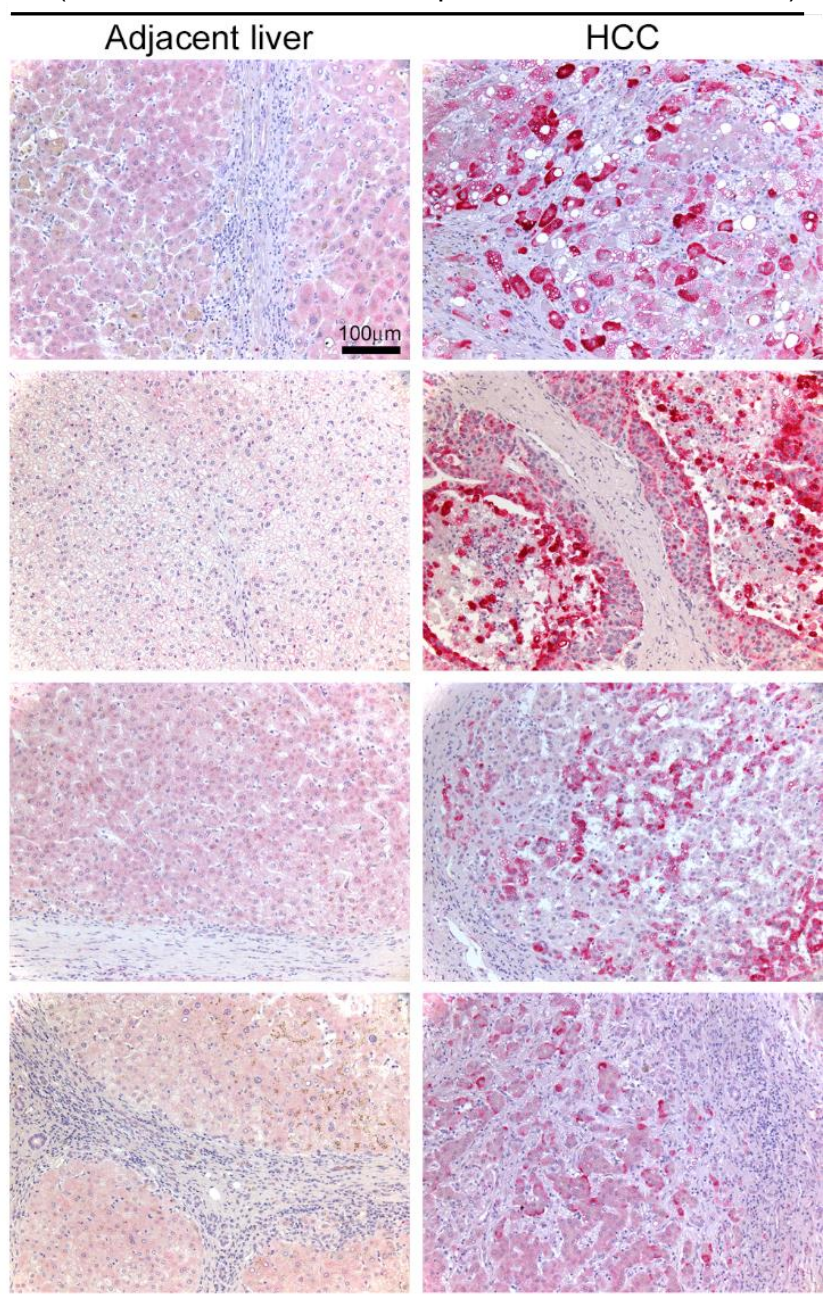

D

ADAMTSL5

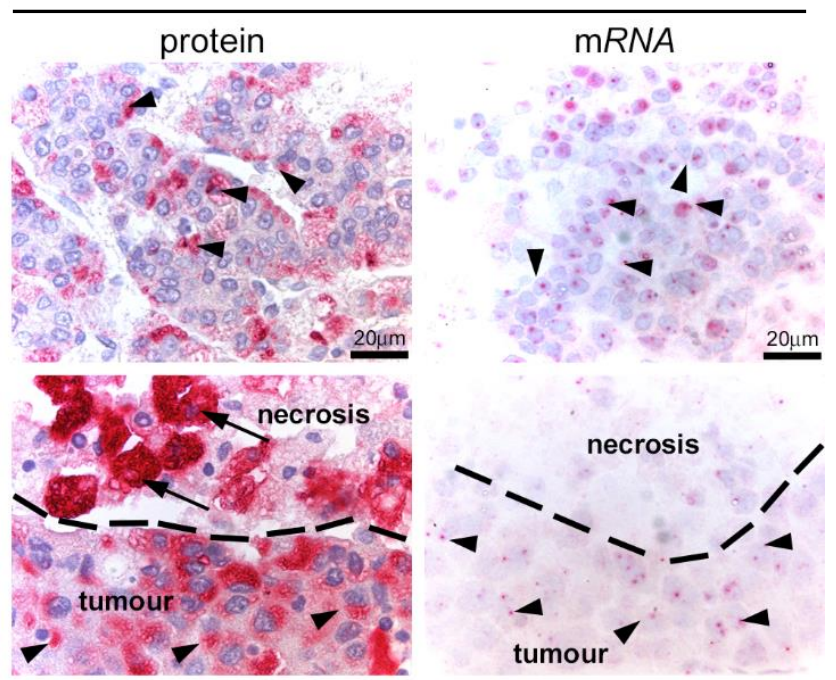

Figure 3 
A

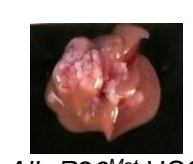

Alb-R26 $6^{\text {Met }} \mathrm{HCC}$
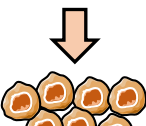

Alb-R26 ${ }^{\text {Met }} \mathrm{HCC}$ cells

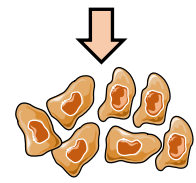

Alb-R26 ${ }^{\text {Met }} \mathrm{HCC}$ shAdamts15 cells
B

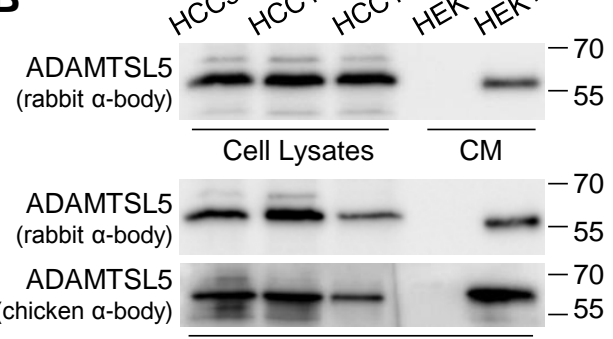

Ponceau

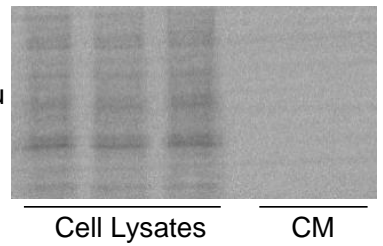

C

$\mathrm{HCC} 13$

shADAMTSL5

cntr sh3.7 sh3.2 sh3.6 sh1.18 $-70$

ADAMTSL5

(rabbit $\alpha$-body)

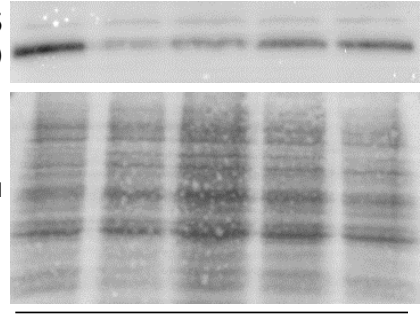

Cell Lysates

ratio ADAMTSL5/ $1 \quad 0,2 \quad 0,2 \quad 0,4 \quad 0,7$

Ponceau
D
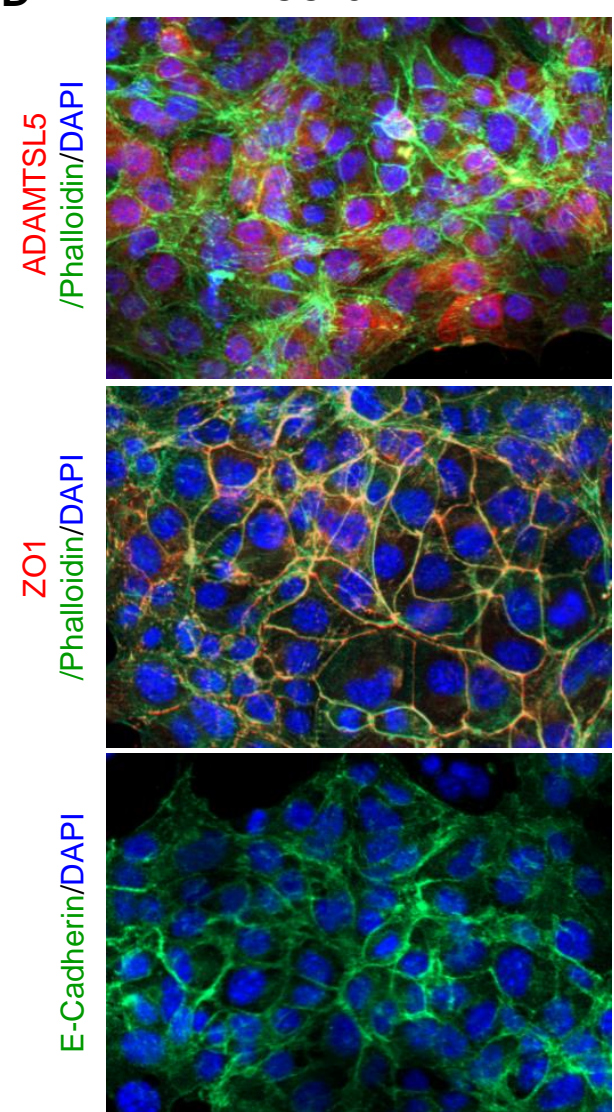

HCC13 3 shAdamtsI5_clone3.7
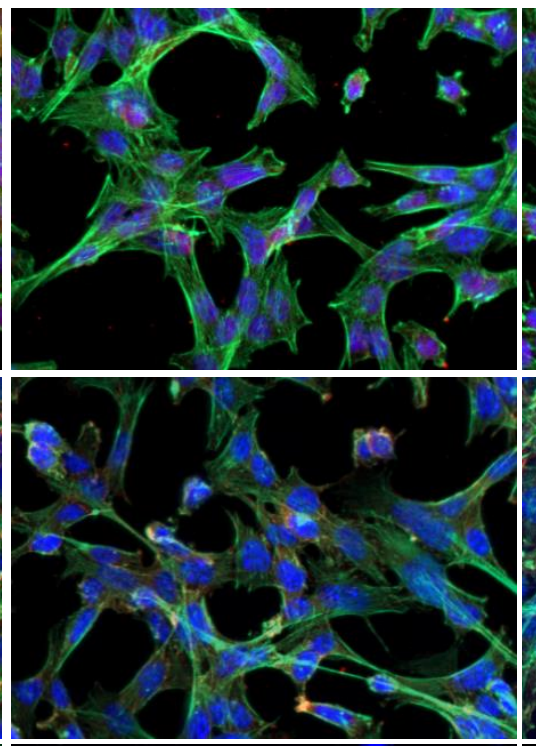

HCC13 3 shAdamtsI5_clone 1.18
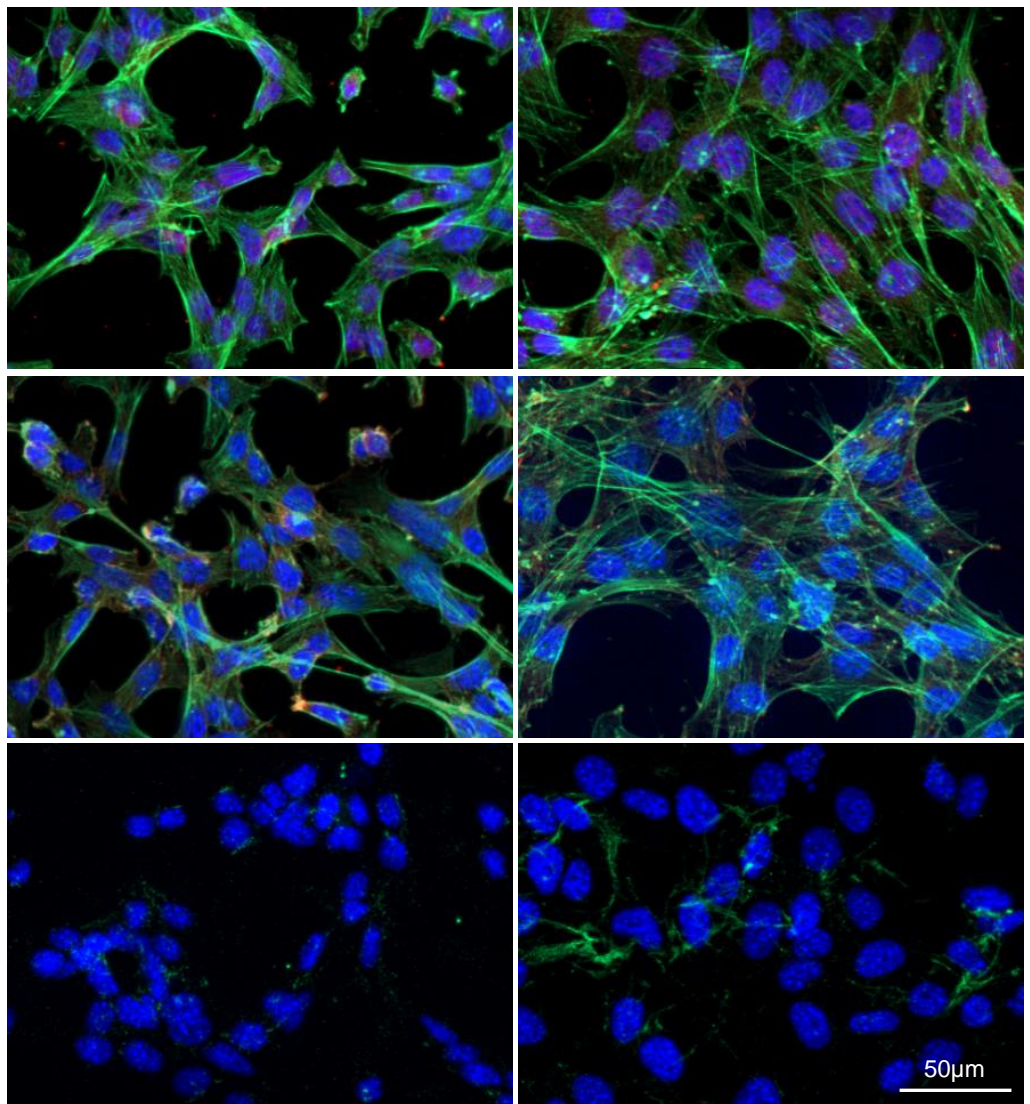

Figure 4 
A
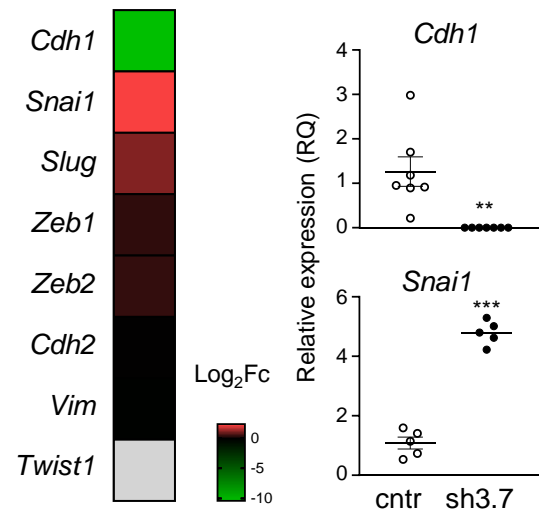

C ${ }^{\alpha-S m a}$

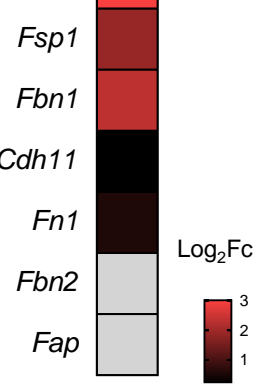

E

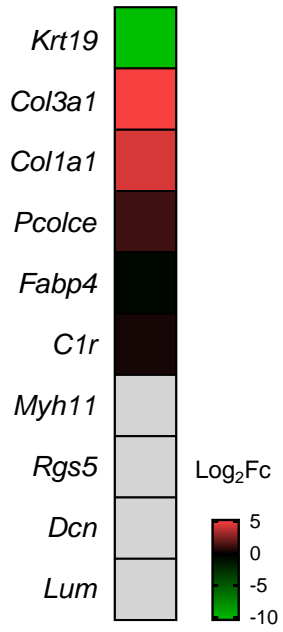

B

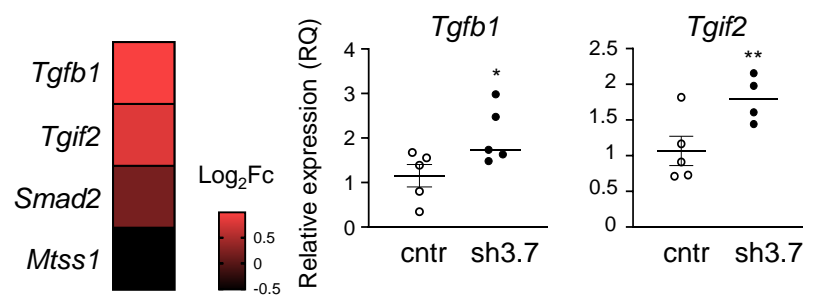

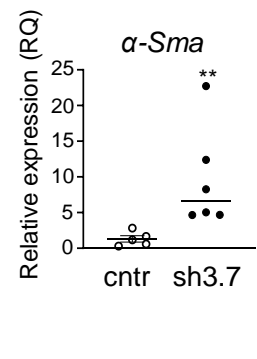
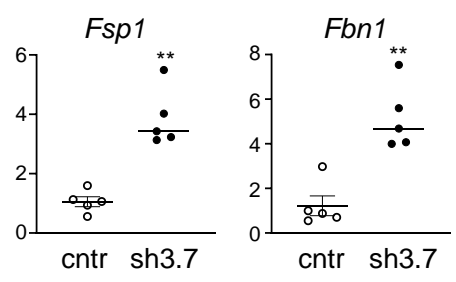

F
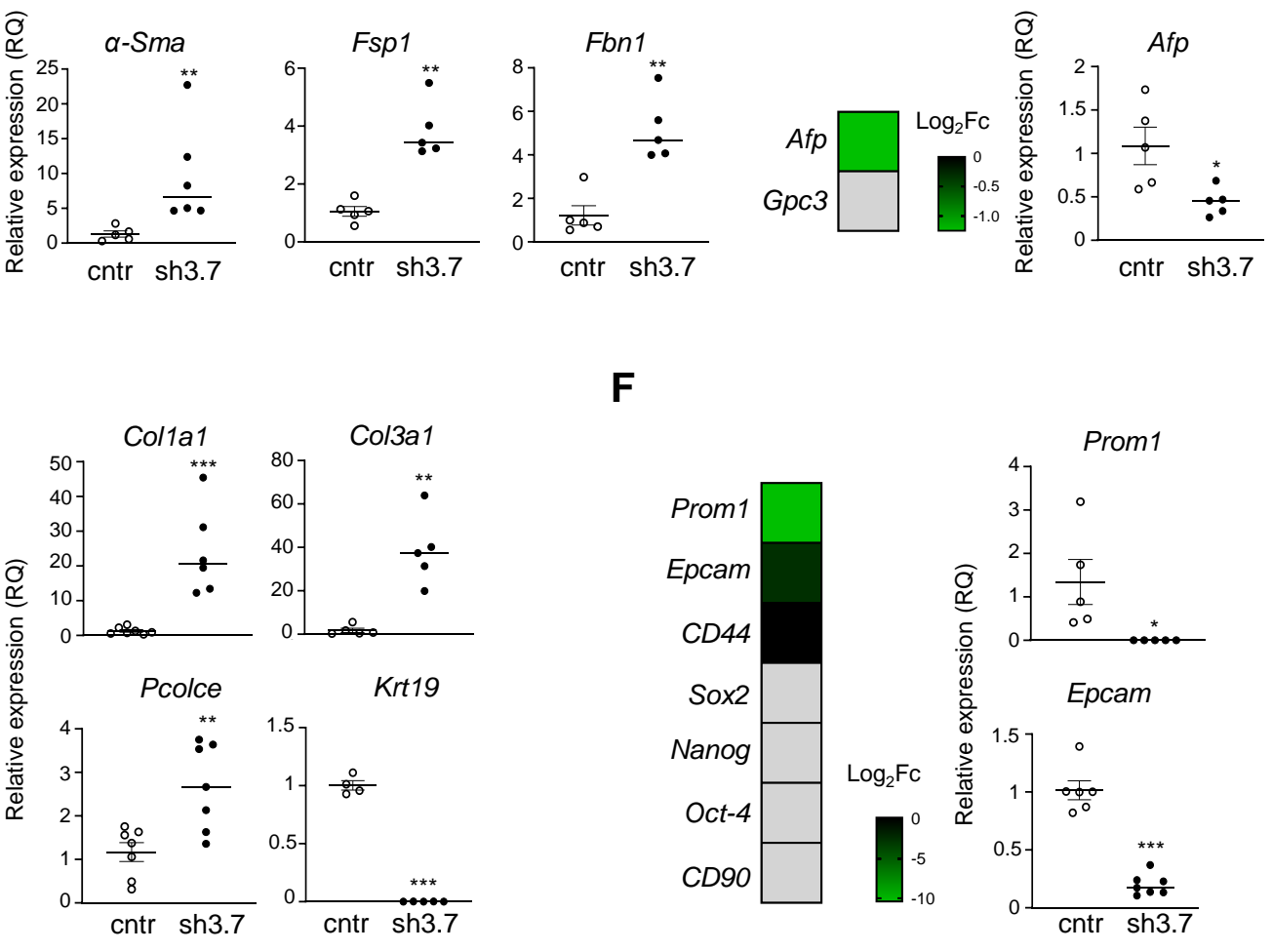

Figure 5

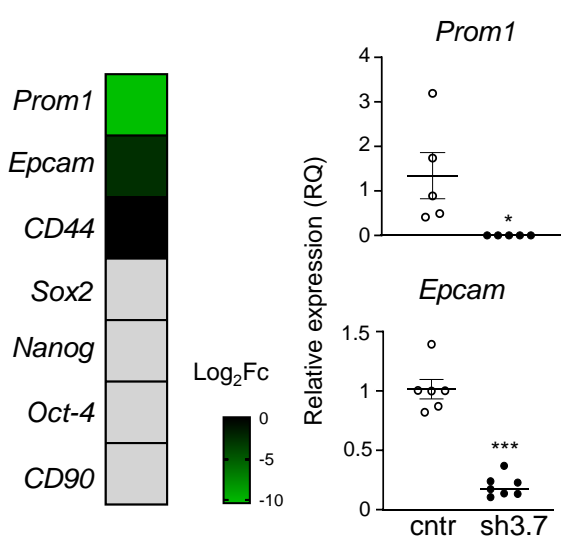


A
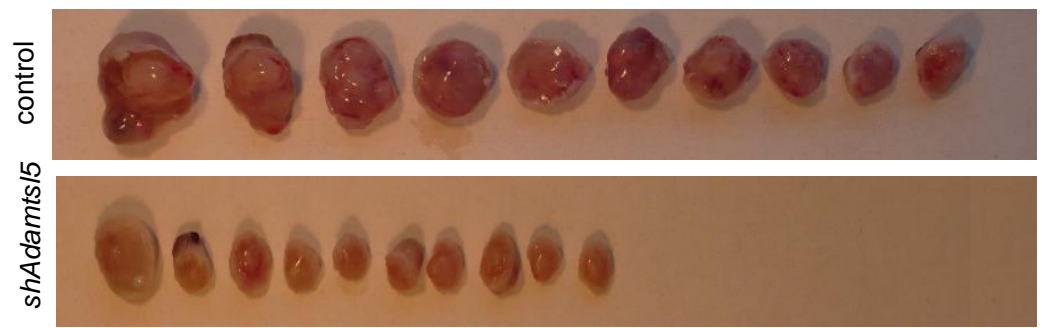

B
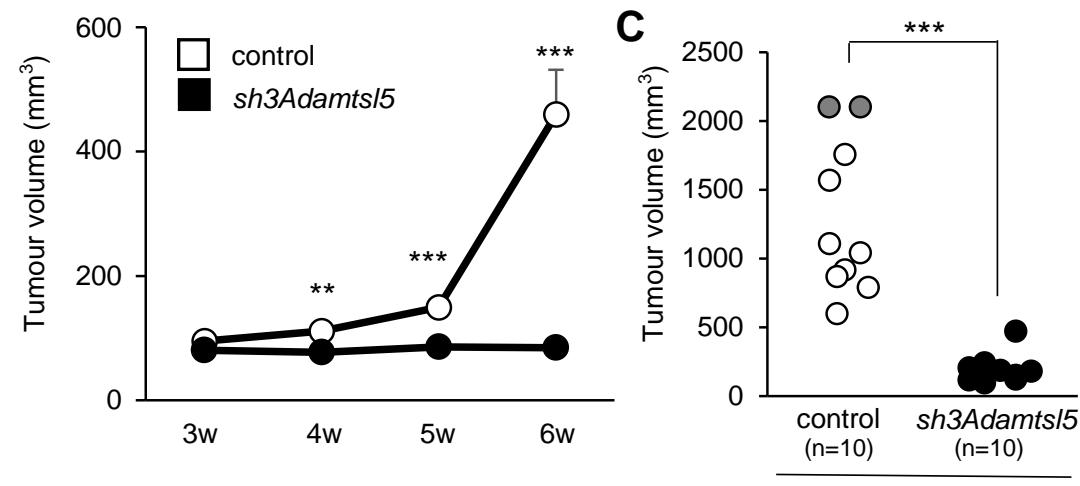

D shAdamtsI5_clonesh3.7

E
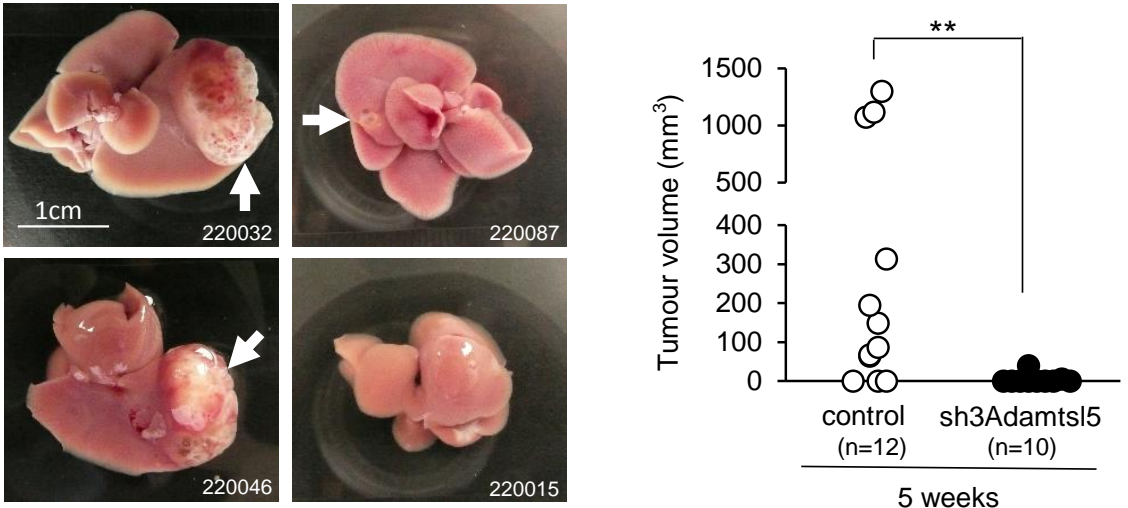

F

SV40 large-T

livers from E15.5 antigen $R 26^{\text {Met }}$ embryos
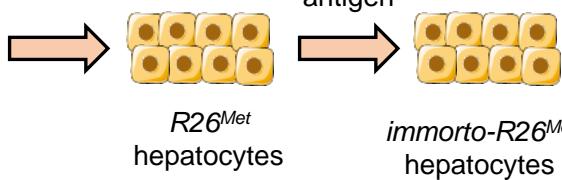

hADAMTSL5

G
immorto-R26 $6^{\text {Met }}$ hepatocytes

immorto-R26Met hepatocytes ${ }^{\text {overADAMTSL5 }}$ immorto- $R 26^{\text {Met }}$ hepatocytes immorto- $R 26^{\text {Met }}$ hepatocytes ${ }^{\text {overADAMTSL5 }}$
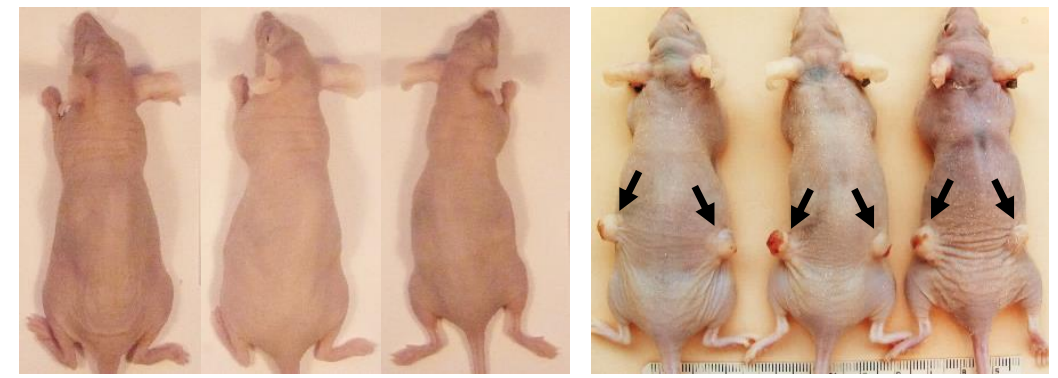

H
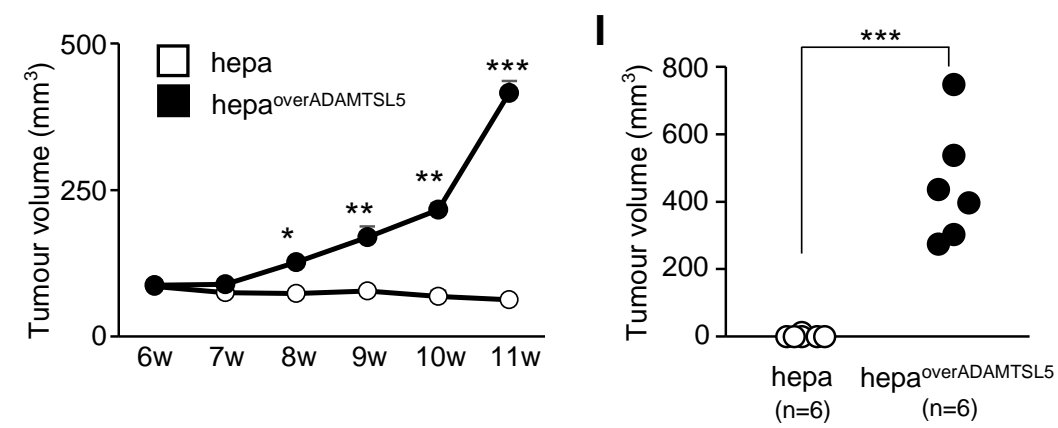

\section{Figure 7}


A

Down-regulated Up-regulated

B shControl shAdamts/5 C
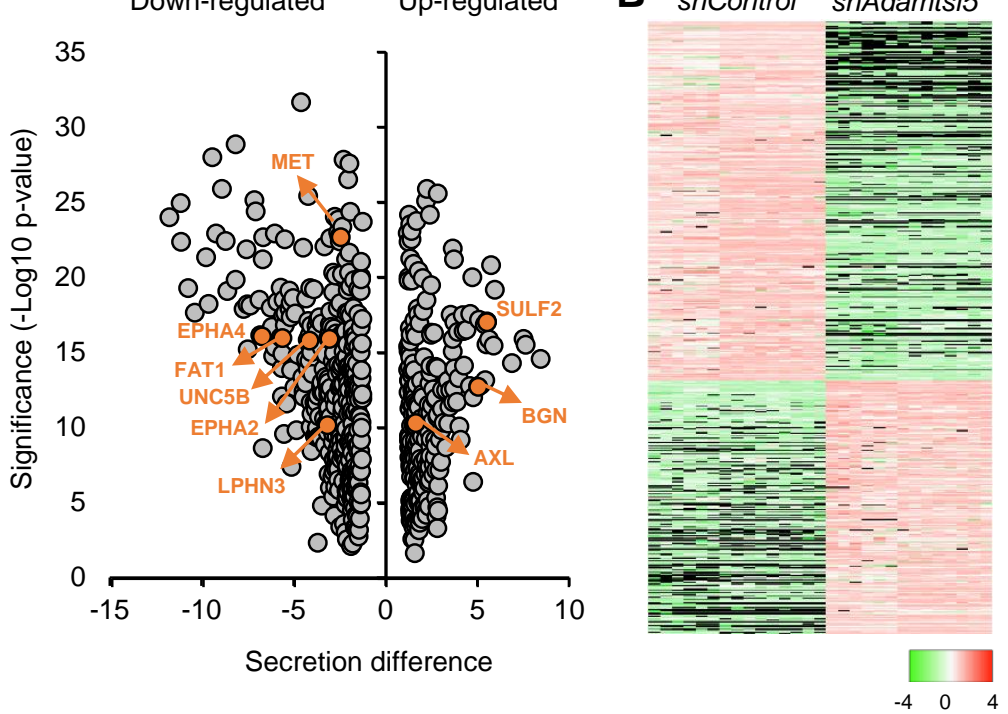

Other glycan degradati Axon guidance Glycosphingolipid biosynthesis Proteoglycans in cancer ECM-receptor interaction Glycosaminoglycan degradation Bacterial invasion of epithelial cells

Mucin type O-glycan biosynthesis AGE-RAGE in diabetic complications Complement and coagulation cascades Amoebiasis

Protein digestion and absorption Pyrimidine metabolism Malaria

Purine metabolism Focal adhesion Thyroid hormone synthesis Pantothenate and CoA biosynthesis One carbon pool by folate

$\begin{array}{lcc}0 & 10 & 20 \\ \text { gnificance } & (-\log 10 & p \text {-value) }\end{array}$

$A X L \quad$ Biglycan Sulf-2

$\mathrm{p}=4,4 \mathrm{E}-11 \quad 35] \quad \mathrm{p}=1,7 \mathrm{E}-13 \quad 30 \quad \mathrm{p}=8,5 \mathrm{E}-18$
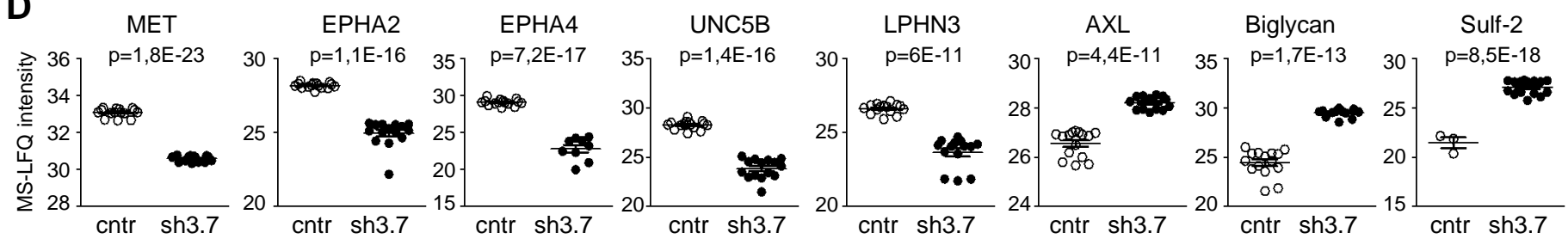

E
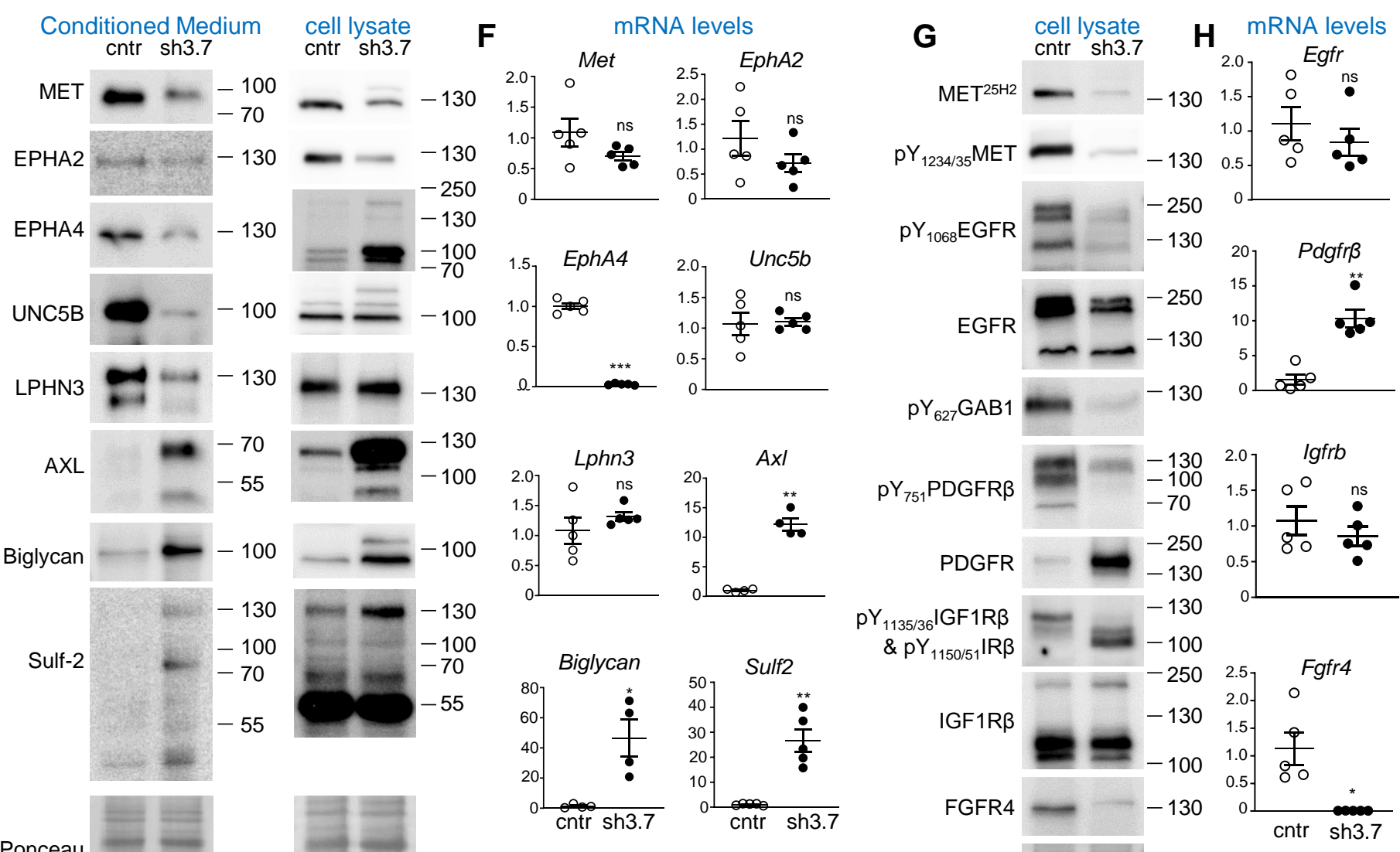

Ponceau

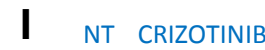

LENVATINIB
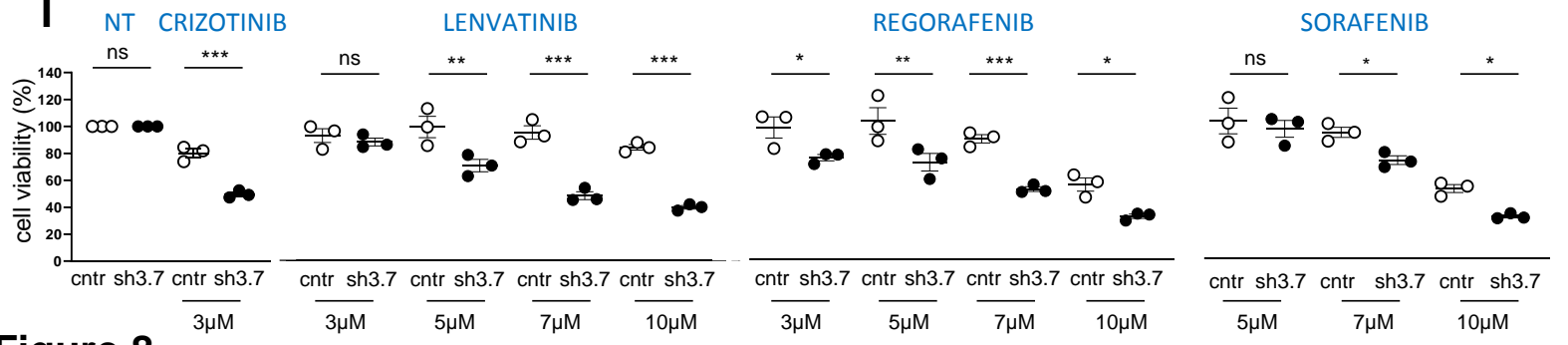

BEMCENTINIB

Figure 8 $\overline{3 \mu \mathrm{M}} \quad \overline{3 \mu \mathrm{M}} \quad \overline{5 \mu \mathrm{M}} \quad \overline{7 \mu \mathrm{M}} \overline{10 \mu \mathrm{M}}$

$\frac{0+0}{0}$

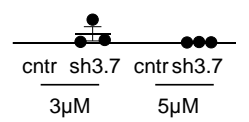




\section{Highlights}

- ADAMTSL5 overexpression in HCC is associated with gene body CGI hypermethylation

- ADAMTSL5 is strongly expressed in a large fraction of human HCC

- $\quad$ Targeting ADAMTSL5 diminishes RTK inputs and interferes with tumorigenicity

- ADAMTSL5 confers tumorigenicity to sensitized, non-transformed liver cells

- $\quad$ Targeting ADAMTSL5 sensitizes HCC cells to drugs currently used in the clinic 